\title{
New Approach to Obtain the Maximum Flow in a Network and Optimal Solution for the Transportation Problems
}

\author{
E. M. U. S. B. Ekanayake ${ }^{1}$, W. B. Daundasekara ${ }^{2}$ \& S. P. C. Perera ${ }^{3}$ \\ ${ }^{1}$ Department of Physical Sciences, Faculty of Applied Sciences, Rajarata University of Sri Lanka, Mihinthale, Sri \\ Lanka \\ ${ }^{2}$ Departments of Mathematics, Faculty of Science, University of Peradeniya, Sri Lanka \\ ${ }^{3}$ Departments of Engineering Mathematics, Faculty of Engineering, University of Peradeniya, Sri Lanka \\ Correspondence: E. M. U. S. B. Ekanayake, Department of Physical Sciences, Faculty of Applied Sciences, \\ Rajarata University of Sri Lanka, Mihinthale, Sri Lanka.
}

Received: June 14, 2021

Accepted: January 17, 2022

Online Published: January 21, 2022

doi:10.5539/mas.v16n1p30

URL: https://doi.org/10.5539/mas.v16n1p30

\begin{abstract}
The maximum flow problem is also one of the highly regarded problems in the field of optimization theory in which the objective is to find a feasible flow through a flow network that obtains the maximum possible flow rate from source to sink. The literature demonstrates that different techniques have been developed in the past to handle the maximum amount of flow that the network can handle. The Ford-Fulkerson algorithm and Dinic's Algorithm are the two major algorithms for solving these types of problems. Also, the Max-Flow Min-Cut Theorem, the Scaling Algorithm, and the Push-relabel maximum flow algorithm are the most acceptable methods for finding the maximum flows in a flow network. In this novel approach, the paper develops an alternative method of finding the maximum flow between the source and target nodes of a network based on the "max-flow." Also, a new algorithmic approach to solving the transportation problem (minimizing the transportation cost) is based upon the new maximum flow algorithm. It is also to be noticed that this method requires a minimum number of iterations to achieve optimality. This study's algorithmic approach is less complicated than the well-known meta-heuristic algorithms in the literature.
\end{abstract}

Keywords: maximum flow, network flow models, transportation problem, initial feasible solution, optimal solution

\section{Introduction}

The flow network is a directed graph where $\mathbf{V}$ is an $\mathbf{n}$-set of nodes that get a flow and $\mathrm{E}$ is an $\mathbf{m}$-set of directed edges that have a capacity. The proportion of flow on an edge can't surpass the limit of the edge. In these situations, it is normally needed to move the maximum amount of flow from a beginning point $\mathbf{s}$ (called the source) to a terminal point $\mathbf{t}$ (called the sink) (Dash, 2019; Dimitri, 1998). We are now interested in related problems that are called maximum flow problems. Each has a nonnegative capacity (weight or time), determining the maximum number of flow units that can pass along the arc.

The network flow problem is one of the most fundamental problems in operations research. Many practical problems can be expressed as network flow problems. Such as modeling traffic in a road system, fluid in pipes, computer networks, current in an electrical circuit, etc. Networks can be broadly classified into two types:

i. Ordinary network flow models require flow protection on all arcs: the measure of flow entering an arc equals the measure of flow leaving the arc.

ii. Generalized network flow models: Generalized networks allow modeling of conditions including inefficient or super-efficient flows as well as flow transformation. For example, transportation problems, assignment problems, maximum value flow, minimum cost flow problems, and shortest path problems, etc.

During the Cold War, the US Air Force at that time was very interested in the Soviet train networks. In these reports, the Air Force collected enough information about the train network that they were able to determine how resources could be transported from the Soviet Union to Europe. And what needed to be done to destroy this 
movement of resources. This problem leads to the concept of a flow network. Aviation-based armed forces scientists T. E. Harris and F. S. Ross (1956) published a classified report considering the rail network that linked the Soviet Union's satellites. Harris and Ross, studying the rail network, modeled it as a graph with 44 vertices and 105 edges, representing joins between those locales in the rail network. The system unraveling technique is based on the Ford-Fulkerson max-stream calculation. The network unraveling method is based on the Ford-Fulkerson max-flow algorithm. Ford and Fulkerson (1962) defined flows as an area of operations research. This concept was implemented by many scientists, statisticians, mathematicians, and engineers.

In the previous few years, different types of algorithms for solving this problem have been proposed, for example, the network unraveling technique based on the Ford-Fulkerson max-flow algorithm (1956), A Parallel Ford-Fulkerson Algorithm For Maximum Flow Problem (Harris and Ross, 1956), Maximum Flow Problem (Ahuja, 1989; Karzanova, 1974). The Maximum Flow Network Interdiction Problem (Douglas, 2010), etc. In like manner, transportation problems have been generally considered in Operation Research. It is one of the fundamental problems of the network flow problem (Dinice, 1970; Elias et al., 1956; Fulkerson \& Dantzig, 1955), which is normally used to minimize the transportation cost for ventures with a couple of sources to a couple of objectives.

The fundamental TP was first proposed by F. L. Hitchcock in 1941, and then independently by T. C. Koopmans in 1947, after which the logical solution procedures from the simplex algorithm were further developed (Kulkarni \& Datar, 2010; Mallick et al., 2016), primarily by Dantzig and then by Charnes et al. (1953). The Simplex Method isn't reasonable for the transportation problem, especially for large scale transportation problems, in view of the interesting structure of the model by charges and Cooper (1954) who made the Stepping Stone method. The literature witnesses that different techniques (Ahmedat et al., 2016-2017; Babu at el., 2013; Deshmukh, 2012; Ekanayake, 2020-2021; Girmay, 2013; Hitchcock, 1941; Khan, 2015; Kulkarni, 2010; Pannerselvam, 2010) have been developed in the past to solve the transportation problem. In this study, we examine a novel approach for solving the maximum-flow problems and TP problems (TPMFP), which is based on the use of the Predecessor cost matrix. The proposed algorithm is basic, straightforward, and simple to actualize. This method is also to be noticed that, requires a minimum number of steps to reach optimality as compare the obtained results with the regular methods. Finally, this procedure is outlined with some numerical examples. We present a few new effective algorithms for the summarized maximum flow problem.

\section{The Key Definitions}

Definition 1 (Edmonds, 1972; Fulkerson, 1955). A flow network is a directed graph $G=(V, E)$, with a source $s$ $\in \mathrm{V}$, a sink $\mathrm{t} \in \mathrm{V}$, and each edge $(\mathrm{i}, \mathrm{j}) \in \mathrm{E}$ has a non-negative capacity $\mathrm{c}(\mathrm{i}, \mathrm{j})$.

Definition 2 (Ford, 1962). Given a flow network $G=(V, E)$, a flow in $\mathrm{G}$ is described by a function

$f: V \times V \rightarrow \mathbb{R}$ Satisfying

i. Capacity constraint: $0 \leq f(i, j) \leq c(i, j)$ for each $i, j \in V$.

ii. $\quad$ Skew -symmetry (Anti symmetry): $f(i, j)=-f(j, i)$ for all $i, j \in V$.

iii. Flow conservation:

For each $i, j \in V$, then $\sum_{i \in V} f(i, j)=\sum_{j \in V} f(j, i)$

Flow into $\mathrm{j}$ Flow out of $\mathrm{j}$

Flow value $|f|=\sum_{i \in V} f(i, k)-\sum_{j \in V} f(k, i)$

Definition 3 (Dimitri, 1998). Predecessor cost matrix

The algorithm represents an $n$ - node network as a square matrix with n- rows and n- columns. Entry (i,j) of the matrix gives the weight $\mathrm{w}_{\mathrm{ij}}$ from node $\mathrm{i}$ to node $\mathrm{j}$.

That is, $W=\left(w_{i j}\right)$, where,

$$
\begin{aligned}
& 0 \\
& \text { if } i=j \\
& w_{i j}=\text { The weight of directed edge }(i, j) \quad \text { if } i \neq j \text { and }(i, j) \in E \\
& \infty \\
& \text { if } i \neq j \text { and }(i, j) \notin E
\end{aligned}
$$

Definition 4 (Dimitri, 1998). Flow Capacity Update. (Augmenting path)

We are given a weighted, directed graph $G=(V, E)$.There is a weight of path $(P)$ from $s$ to $t$ for all edges $(i, j) \in P$, sub graph of $G^{\prime}=\left(V^{\prime}, E^{\prime}\right) ; V^{\prime} \subseteq V$ and $E^{\prime} \subseteq E$ s.t. 
i. $\quad E^{\prime} \mathrm{s}$ the rechargeable edges.

ii. $\quad G^{\prime}$ is the path from $s$ to $t$.

iii. For all $v \in V^{\prime}$; the unique direct path from $s$ to $t$.

iv. Max flow-carrying path $\boldsymbol{P}$ has been found from $s$ to $t\left(w^{*}{ }_{i j}>0\right.$ for every arc $(i, j)$ on the path), and $w_{s t}{ }^{(m)}=\operatorname{Min}\left\{w^{*}{ }_{i j} \mid:(i, j)\right.$ on $\left.\boldsymbol{P}\right\}$, where $m$ is number of iteration.

v. The capacity constraints are satisfied

a. $\operatorname{If}(i, j) \in \boldsymbol{P}$, then $w(i, j)-w_{s t}{ }^{(m)}$

b. Otherwise, $(i, j) \notin \boldsymbol{P}$ is from the original flow.

\section{Definition 5.}

Network flow algorithms depend on the idea of augmenting paths (predecessor cost matrix) and more than once find a path of positive capacity from $s$ to $t$ and add it to the flow. It tends to be demonstrated that the flow through a network is optimal if and only if it contains no augmenting paths.

Definition 6 (Dimitri, 1998).

The value of a Max flow is defined as $|w|=\sum_{(s, t) \in V} w_{s t}{ }^{(m)}$ which is the total amount out of the source (also equal the total amount into the sink).

\section{Mathematical Model}

\subsection{Max Flow}

The problem can be formulated as follows;

$$
\text { Maximize } \quad V
$$

Subject to;

$$
\begin{gathered}
\sum_{j} x_{i j}-\sum_{k} x_{k i}=\left\{\begin{aligned}
V & \text { if } i=s \\
-V & \text { if } i=t \\
0 & \text { Otherwise }
\end{aligned}\right. \\
0 \leq x_{i j} \leq u_{i j} ;=1,2, \ldots \text { m and } j=1,2,3 . . n
\end{gathered}
$$

We assume that there is no arc from $t$ to $s$ and $u_{i j}=\infty$ if arc $i$ to $j$ has unlimited capacity.

3.1.1 Predecessor Cost Matrix for Max Flow

Table 1. Predecessor cost matrix for Max Flow

\begin{tabular}{lccccccc}
\hline & $\mathrm{A}$ & $\mathrm{B}$ & $\mathrm{C}$ & $\ldots$. & $\mathrm{L}$ & $\mathrm{M}$ & $\mathrm{N}$ \\
\hline $\mathrm{A}$ & 0 & $\alpha_{a b}$ & $\alpha_{a c}$ & $\ldots$. & $\alpha_{a l}$ & $\alpha_{a m}$ & $\alpha_{a n}$ \\
$\mathrm{~B}$ & $\alpha_{b a}$ & 0 & $\alpha_{b c}$ & $\ldots$. & $\alpha_{b l}$ & $\alpha_{b m}$ & $\alpha_{b n}$ \\
$\mathrm{C}$ & $\alpha_{c a}$ & $\alpha_{c b}$ & 0 & $\ldots$. & $\alpha_{c l}$ & $\alpha_{c m}$ & $\alpha_{c n}$ \\
$\vdots$ & $\vdots$ & $\vdots$ & $\vdots$ & $\ldots$. & $\vdots$ & $\vdots$ & $\vdots$ \\
$\mathrm{L}$ & $\alpha_{l a}$ & $\alpha_{l b}$ & $\alpha_{l c}$ & $\ldots$. & 0 & $\alpha_{l m}$ & $\alpha_{l n}$ \\
$\mathrm{M}$ & $\alpha_{m a}$ & $\alpha_{b m}$ & $\alpha_{m c}$ & $\ldots$. & $\alpha_{m l}$ & 0 & $\alpha_{m n}$ \\
$\mathrm{~N}$ & $\alpha_{n a}$ & $\alpha_{b n}$ & $\alpha_{n c}$ & $\ldots$. & $\alpha_{n l}$ & $\alpha_{n m}$ & 0 \\
\hline
\end{tabular}

Where

$$
\alpha_{i j}=\left\{\begin{array}{c}
\delta ; \text { real number } \\
\infty ; \text { Otherwise }
\end{array}\right.
$$

For $i, j=\mathrm{A}, \mathrm{B}, \mathrm{C} \ldots \ldots \mathrm{L}, \mathrm{M}, \mathrm{N}$.

\subsection{Transportation Problem (Rayand \& Hosain, 2007)}

The total transportation cost is

$$
\operatorname{Minimize} \sum_{i=1}^{m} \sum_{j=1}^{n} X_{i j} C_{i j}
$$

Subject to the constraints

$$
\begin{aligned}
\text { i. } & \sum_{j=1}^{n} X_{i j}=a_{i}, i=1,2, \ldots, m \\
\text { ii. } & \sum_{i=1}^{m} X_{i j}=b_{j}, \quad j=1,2, \ldots, n \text { and }
\end{aligned}
$$


iii. $\quad X_{i j} \geq 0$ for all $i=1,2, \ldots, m$ and $j=1,2, \ldots, n$

Note that here the sum of the supplies equals the sum of the demands. i.e. $\sum_{i=1}^{m} a_{i}=\sum_{j=1}^{n} b_{j}$. Such problems are called balanced transportation problems and otherwise, i.e. $\sum_{i=1}^{m} a_{i} \neq \sum_{j=1}^{n} b_{j}$, known as unbalanced transportation problems.

$$
\begin{array}{ll}
\text { i. } & \sum_{i=1}^{m} a_{i}>\sum_{j=1}^{n} b_{j} \\
\text { ii. } & \sum_{i=1}^{m} a_{i}<\sum_{j=1}^{n} b_{j}
\end{array}
$$

Introduce a dummy origin in the transportation table; the cost associated with this origin is set equal to zero. The availability at this origin is: $\sum_{i=1}^{m} a_{i}-\sum_{j=1}^{n} b_{j}=0$.

\subsubsection{Predecessor Cost Matrix for TP}

\begin{tabular}{|c|c|c|c|c|c|c|c|c|c|c|c|}
\hline & $S_{1}$ & $S_{2}$ & ..... & $S_{m-1}$ & $S_{m}$ & $D_{1}$ & $D_{2}$ & $\cdots$ & $D_{n-1}$ & $D_{n}$ & \\
\hline$S_{1}$ & 0 & $\infty$ & & $\infty$ & $\infty$ & $C_{11}$ & $C_{12}$ & $\ldots$ & $C_{1(n-1)}$ & $C_{1 n}$ & $\overline{a_{1}}$ \\
\hline$S_{2}$ & & 0 & $\ldots$. & $\infty$ & $\infty$ & $C_{21}$ & $C_{22}$ & $\ldots$ & $C_{2(n-1)}$ & $C_{2 n}$ & $a_{2}$ \\
\hline$\vdots$ & $\vdots$ & $\vdots$ & & $\vdots$ & $\vdots$ & $\vdots$ & $\vdots$ & $\ldots$ & $\vdots$ & $\vdots$ & $\vdots$ \\
\hline$S_{m-1}$ & $\infty$ & $\infty$ & $\cdots$ & 0 & $\infty$ & $C_{(m-1) 1}$ & $C_{(m-1) 2}$ & $\cdots$ & $C_{(m-1)(n-1)}$ & $C_{(m-1) n}$ & $a_{m-1}$ \\
\hline$S_{m}$ & $\infty$ & $\infty$ & $\ldots$. & $\infty$ & 0 & $C_{m 1}$ & $C_{m 2}$ & $\ldots$ & $C_{m(n-1)}$ & $C_{m n}$ & $a_{m}$ \\
\hline$D_{1}$ & $C_{11}$ & $C_{21}$ & & $C_{(m-1) 1}$ & $C_{m 1}$ & 0 & $\infty$ & $\ldots$. & $\infty$ & $\infty$ & $b_{1}$ \\
\hline$D_{2}$ & $C_{12}$ & $C_{22}$ & & $C_{(m-1) 2}$ & $C_{m 2}$ & $\infty$ & 0 & $\ldots$. & $\infty$ & $\infty$ & $b_{2}$ \\
\hline$\vdots$ & & & & & & $\vdots$ & $\vdots$ & $\ldots$ & $\vdots$ & $\vdots$ & \\
\hline$D_{n-1}$ & $C_{1(n-1)}$ & $C_{2(n-1)}$ & & $C_{(m-1)(n-1)}$ & $C_{(m-1)(n-1)}$ & $\infty$ & $\infty$ & $\cdots$ & 0 & $\infty$ & $b_{n-1}$ \\
\hline \multirow[t]{2}{*}{$D_{n}$} & $C_{1 n}$ & $C_{2 n}$ & & $C_{(m-1) n}$ & $C_{(m-1) n}$ & $\infty$ & $\infty$ & $\ldots$. & $\infty$ & 0 & $b_{n}$ \\
\hline & $b_{1}$ & $b_{2}$ & & $b_{n-1}$ & $b_{n}$ & $a_{1}$ & $a_{2}$ & & $a_{m-1}$ & $a_{m}$ & \\
\hline
\end{tabular}

Table 2. Predecessor cost matrix for TP

\section{Illustration of the Proposed Algorithm}

A wide range of decision problems that can be modeled as network optimization problems and solved efficiently and effectively can be solved using network models. Some of these decision problems, such as transportation or commodity flow, are physical in nature. On the other hand, many network problems, such as the critical path activity network in project management, are abstract representations of processes or activities. Researchers have improved a number of algorithms for solving maximal-flow problems over the last fifty years. In this algorithm, we presented a new algorithm for calculating the maximum flow in a network. The new maximum flow algorithm also serves as the foundation for a new algorithmic approach to solving the transportation problem (minimizing transportation costs).

\subsection{New Algorithm}

Step 1. Construct the Predecessor cost matrix

Step 2. Start with $1^{\text {st }}$ row (source nodes) and select any weighted node $k$ with $w^{*}{ }_{s k}>0$.

Step 3. Next Select $\mathrm{k}^{\text {th }}$ row and select any another weighted node $i\left(w^{*}{ }_{k i}\right)$, after that select $\mathrm{i}^{\text {th }}$ row and select any weighted node $j\left(w^{*}{ }_{i j}\right)$, next select $\mathrm{j}^{\text {th }}$ row and select any weighted node $j\left(w^{*}{ }_{j h}\right)$, and label every node $h$ with $w^{*}{ }_{j h}>0$ until either sink $t$ has been labeled $\left(w^{*}{ }_{. t}\right)$. (There is a path $(\boldsymbol{P})$ from $\boldsymbol{s}$ to $\boldsymbol{t}$ )

Step 4. Find the max flow $w_{s t}{ }^{(m)}=\operatorname{Min}\left\{w^{*}{ }_{i j} \mid:(i, j)\right.$ on $\left.\boldsymbol{P}\right\}$

Step 5. Flow is updated (Definition 4)

Step 6. If the $w^{*}{ }_{s k}=0(k=2,3,4,,, n)$ or node k with $w^{*}{ }_{k . .}=0$, yes go to Step 7, no go to Step 3 .

Step 7. Then terminate.

\subsection{Available Algorithm (Dash at el., 2019)}

Proposed Algorithm

The proposed algorithm is given below:

Step 1: first initialize the flow $\mathrm{f}$ to 0 , for each edge (u, v) $\in \mathrm{E}[\mathrm{G}]$,

Step2: $\mathrm{f}(\mathrm{u}, \mathrm{v})=\mathrm{f}(\mathrm{v}, \mathrm{u})=0$ 
Step 3: Calculate maximum capacity $\mathrm{C}$ in the flow network and then calculate $D=11^{\log _{11} C}$

Step 4: while $\mathrm{D} \geq 1$

Step 5: If there exists an augmenting path $\mathrm{p}$ from $\mathrm{s}$ to $t$ in the residual network $G_{f}$ with capacity at least $\mathrm{D}$ the select it and go to step 6; otherwise go to step 9 .

Step6: set $c_{f}(\mathrm{p})=\min \left\{c_{f}(\mathrm{u}, \mathrm{v}):(\mathrm{u}, \mathrm{v})\right.$ is in $\left.\mathrm{p}\right\}$

Step 7: For each $(\mathrm{u}, \mathrm{v}) \in \mathrm{p}$, if $(\mathrm{u}, \mathrm{v}) \in \operatorname{E} \operatorname{set} \mathrm{f}(\mathrm{u}, \mathrm{v})=\mathrm{f}(\mathrm{u}, \mathrm{v})+c_{f}(p)$ else $\mathrm{f}(\mathrm{u}, \mathrm{v})=-\mathrm{f}(\mathrm{v}, \mathrm{u})$

Ste $\mathrm{p} 8$ : Calculate the flow value.

Step 9: $\mathrm{D}=\frac{D}{11}$

Step10: The flow is maximum

\subsection{New Algorithm for TP}

Step-1. Construct a Predecessor Transportation Cost Table (PTCT) from the given transportation problem.

Step-2. Ensure whether the PTCT is balanced or not, if not, make it balanced and complete PTCT of order $m \times n$.

Step 3. Calculate, $\varnothing=\frac{\sum_{I=1}^{n} a_{i}}{m+n-1}=\frac{\sum_{I=1}^{n} b_{j}}{m+n-1}$

Step 4. Distinguish the source or Demand cell close $\emptyset$.

Step 5. $\varnothing$ cell placed at the starting nodes with the minimum $C_{i j}$ value of the PTCT to make the first allocation Step 6. Next utilizing the above Algorithm and goes to head.

Step 7. Then terminate and calculate the total transportation cost

\section{A Comparison of the Methods}

\subsection{Comparative Assessment with Max Flow}

A numerical example has been unraveled for finding the maximum value of a Maximum flow problem by utilizing proposed algorithm which is given underneath (Dash et al., 2019).

Consider a pipeline system in an Azimpur colony to supply gas in different zones of an Azimpur Colony of Dhaka in the Bangladesh. The pipeline has a communicated capacity in per unit per hour between any two zones which given as a maximum flow at which gas can flow by the pipe between those two zones. Presently, Consider I need to supply gas from the source zone to the sink zone, assume the source zone is say A and the sink zone is $\mathrm{F}$ and gas passes into 4 others zones before getting from source to sink. Suppose B, C, D, E, are of these 4 zones and pipeline between any two zones has indicated capacity. Demonstrates the input information's which have given to the problem talked above in the following Table 1

Table 3. Indicate capacities of each pipeline between two zones

\begin{tabular}{lllllllll}
\hline Source part & A & A & B & B & D & D & E & E \\
Destination part & B & C & D & C & E & F & B & F \\
Capacity(Gallons/hour) & 35 & 10 & 14 & 40 & 19 & 20 & 9 & 25 \\
\hline
\end{tabular}

Calculate the maximum amount of gas which can flow from A to $\mathrm{F}$.

\section{Step 1.}

Table 4. Predecessor cost matrix

\begin{tabular}{rllllllllllllll} 
& & $\mathrm{A}$ & $\mathrm{B}$ & $\mathrm{C}$ & $\mathrm{D}$ & $\mathrm{E}$ & $\mathrm{F}$ & & $\mathrm{A}$ & $\mathrm{B}$ & $\mathrm{C}$ & $\mathrm{D}$ & $\mathrm{E}$ & $\mathrm{F}$ \\
\hline $\mathrm{A}$ & 0 & 35 & $\infty$ & 10 & $\infty$ & $\infty$ & $\mathrm{A}$ & 0 & 21 & $\infty$ & 10 & $\infty$ & $\infty$ \\
$\mathrm{B}$ & $\infty$ & 0 & 14 & 40 & $\infty$ & $\infty$ & $\mathrm{B}$ & 14 & 0 & 0 & 40 & $\infty$ & $\infty$ \\
$\mathrm{C}$ & $\infty$ & $\infty$ & 0 & $\infty$ & 19 & 20 & $\mathrm{C}$ & $\infty$ & 14 & 0 & $\infty$ & 19 & 6 \\
$\mathrm{D}$ & $\infty$ & $\infty$ & $\infty$ & 0 & 35 & $\infty$ & $\mathrm{D}$ & $\infty$ & $\infty$ & $\infty$ & 0 & 35 & $\infty$ \\
$\mathrm{E}$ & $\infty$ & 9 & $\infty$ & $\infty$ & 0 & 25 & $\mathrm{E}$ & $\infty$ & 9 & $\infty$ & $\infty$ & 0 & 25 \\
$w_{A F}{ }^{(m)}=\operatorname{Min}\left\{w^{*}{ }_{i j} \mid:(i, j)\right.$ & on $\boldsymbol{P}\}$ & $\infty$ & $=$ Min $\{35,14,20\}=14$ & path A-B-C-F & & & &
\end{tabular}




\section{Step 2.}

Table 5. Predecessor cost matrix

\begin{tabular}{|c|c|c|c|c|c|c|c|c|c|c|c|c|c|}
\hline & A & B & $\mathrm{C}$ & $\mathrm{D}$ & E & $\mathrm{F}$ & & A & B & $\mathrm{C}$ & D & $\mathrm{E}$ & $\mathrm{F}$ \\
\hline A & 0 & 21 & $\infty$ & 10 & $\infty$ & $\infty$ & $\mathrm{A}$ & 0 & 0 & $\infty$ & 10 & $\infty$ & $\infty$ \\
\hline B & 14 & 0 & 0 & 40 & $\infty$ & $\infty$ & B & $14+21$ & 0 & 0 & 19 & $\infty$ & $\infty$ \\
\hline $\mathrm{C}$ & $\infty$ & 14 & 0 & $\infty$ & 19 & 6 & $\mathrm{C}$ & $\infty$ & 14 & 0 & $\infty$ & 19 & 6 \\
\hline D & $\infty$ & $\infty$ & $\infty$ & 0 & 35 & $\infty$ & $\mathrm{D}$ & $\infty$ & 21 & $\infty$ & 0 & 16 & $\infty$ \\
\hline $\mathrm{E}$ & $\infty$ & 9 & $\infty$ & $\infty$ & 0 & 25 & $\mathrm{E}$ & $\infty$ & 9 & $\infty$ & 21 & 0 & 4 \\
\hline $\mathrm{F}$ & $\infty$ & $\infty$ & 14 & $\infty$ & $\infty$ & 0 & $\mathrm{~F}$ & $\infty$ & $\infty$ & 14 & $\infty$ & 21 & 0 \\
\hline
\end{tabular}

Step 3.

Table 6. Predecessor cost matrix

\begin{tabular}{|c|c|c|c|c|c|c|c|c|c|c|c|c|c|c|c|}
\hline & & A & B & $\mathrm{C}$ & $\mathrm{D}$ & $\mathrm{E}$ & $\mathrm{F}$ & & & A & B & $\mathrm{C}$ & $\mathrm{D}$ & $\mathrm{E}$ & $\mathrm{F}$ \\
\hline A & $\mathrm{S}$ & 0 & 0 & $\infty$ & 10 & $\infty$ & $\infty$ & A & $\mathrm{S}$ & 0 & 0 & $\infty$ & 6 & $\infty$ & $\infty$ \\
\hline B & 1 & $14+21$ & 0 & 0 & 19 & $\infty$ & $\infty$ & B & 1 & $14+21$ & 0 & 0 & 19 & $\infty$ & $\infty$ \\
\hline $\mathrm{C}$ & 2 & $\infty$ & 14 & 0 & $\infty$ & 19 & 6 & $\mathrm{C}$ & 2 & $\infty$ & 14 & 0 & $\infty$ & 19 & 6 \\
\hline D & 3 & $\infty$ & 21 & $\infty$ & 0 & 16 & $\infty$ & $\mathrm{D}$ & 3 & $\infty$ & $21+4$ & $\infty$ & 0 & 12 & $\infty$ \\
\hline $\mathrm{E}$ & 4 & $\infty$ & 9 & $\infty$ & 21 & 0 & 4 & $\mathrm{E}$ & 4 & $\infty$ & 9 & $\infty$ & $21+4$ & 0 & 0 \\
\hline $\mathrm{F}$ & $\mathrm{t}$ & $\infty$ & $\infty$ & 14 & $\infty$ & 21 & 0 & $\mathrm{~F}$ & $\mathrm{t}$ & $\infty$ & $\infty$ & 14 & $\infty$ & $21+4$ & 0 \\
\hline
\end{tabular}

Now we construct the following table to compare different algorithms with our proposed algorithm

Table 7. Assessment of the residual obtained by different methods

\begin{tabular}{lll}
\hline Name of Algorithm & Number of Iterations & Number of augmentation \\
\hline Ford -Fulkerson & 4 & 4 \\
Edmonds-Karp & 3 & 3 \\
Md.Al-Amin Khan & 4 & 3 \\
Faruque Ahmed & 3 & 3 \\
Modified Edmonds-Karp & 3 & 3 \\
Protima Dash et.al.Method & 2 & 3 \\
New Method & 2 & 3
\end{tabular}

By implies new algorithm yields better outcomes that will be resulted in maximum flow in a network flow problem involving a fewer number of iterations just as fewer augmentations of this we needed to propose.

\subsection{Comparative Assessment with TP}

This section gives execution relationships over the distinctive eminent methods - NWCR, LCM, VAM, New method (TPMFP) and to find the optimal result by the solutions obtained from disparate problems. Comparable assessments are performed and illustrated in the immediately following sections. The detailed representation of the numerical information of Table is given in Appendix A. 
Table 8. A comparative results obtained by NWCM, LCM, VAM, IAM and New method for the FIVE unbalance transportation problem.

\begin{tabular}{llllllllllll}
\hline $\begin{array}{l}\text { Problem chosen from } \\
\text { Ahmed et al. }\end{array}$ & \multicolumn{3}{c}{$\begin{array}{l}\text { TCIFS } \\
\left(\boldsymbol{I}_{\boldsymbol{F} S}\right)\end{array}$} & \multicolumn{3}{c}{$\begin{array}{l}\text { Optimal } \\
\text { result }\left(\boldsymbol{O}_{\boldsymbol{c}}\right)\end{array}$} & \multicolumn{3}{c}{ Percentage of Deviation from optimal solution } \\
\hline & NWCM & $\mathbf{L C M}$ & VAM & IAM & TPMFP & & NWCM & LCM & VAM & IAM & TPMFP \\
UBTP-1. & 1,815 & 1,185 & 1,745 & 1,695 & 1,655 & 1,650 & 9.69 & 14.24 & 5.75 & 2.72 & 0.30 \\
UBTP-2. & 18,800 & 8,800 & 8,350 & 8,400 & 7,750 & 7,750 & 142.6 & 13.54 & 7.74 & 8.38 & 0.00 \\
UBTP-3. & 14,725 & 14,625 & 13,225 & 13,075 & 12,475 & 2,475 & 18.03 & 17.23 & 6.01 & 4.80 & 0.00 \\
UBTP-4. & 13,100 & 9,800 & 9,200 & 9,200 & 9,200 & 9,200 & 42.39 & 6.52 & 0.00 & 0.00 & 0.00 \\
UBTP-5. & 8,150 & 6,450 & 6,000 & 5,850 & 5,600 & 5,600 & 45.53 & 15.17 & 7.14 & 4.46 & 0.00 \\
\hline
\end{tabular}

The TPMFP method's efficiency was also tested by solving five benchmark problems, and performance comparisons between the various well-known methods (NWCM, LCM, VAM, and IAM) are provided in Table 8 . It is clear that the TPMFP method produces a comparatively more appealing result. It can also be demonstrated that the TPMFP provides a motivating initial feasible solution (IFS) by requiring a minimum number of iterations. Table 8 is depicted as a bar graph in Figure 1 and as a line graph in Figure 2 to demonstrate the significance of the proposed method, TPMFP, over other considered existing methods in terms of transportation cost, IFS, and percentage deviation (PD), respectively:

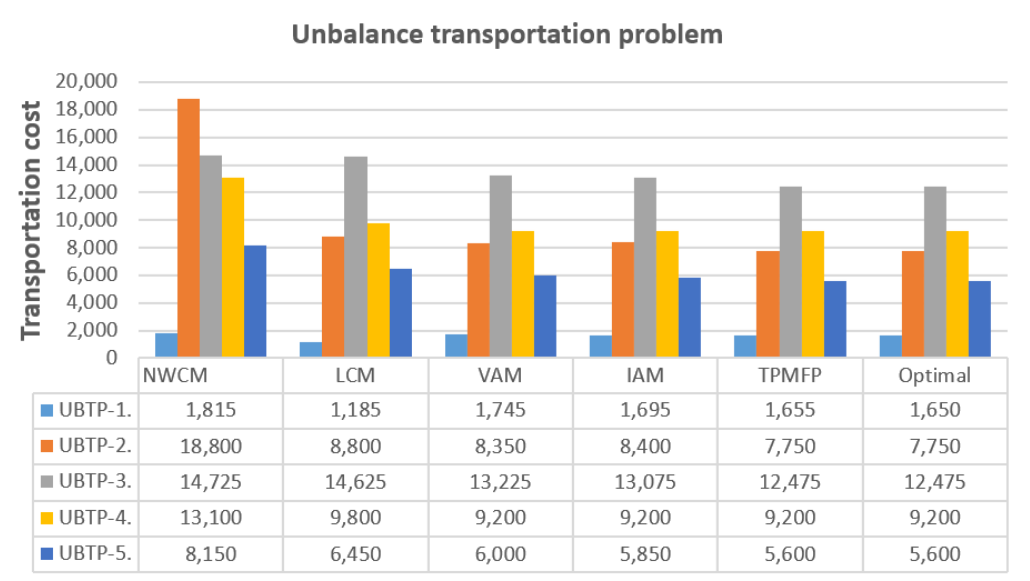

Figure 1. Comparative study of the result obtained by NWCM, LCM, VAM, IAM, and TPMFP

Line graph presented in Figure 2 indicates PD of the NWCM, LCM, VAM, IAM and TPMFP from the minimal total cost solution obtained in Table 8:

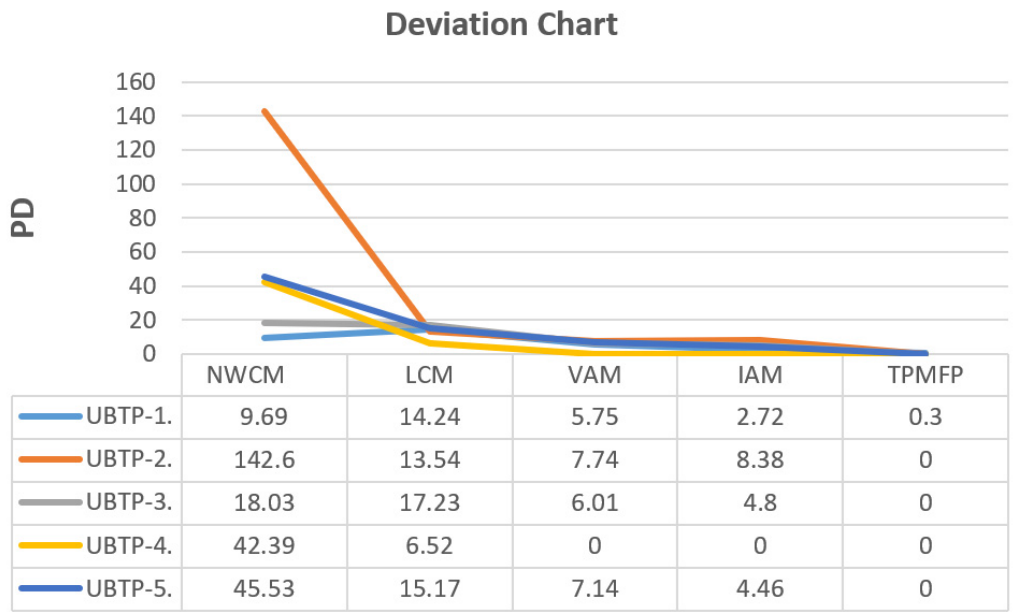

Figure 2. PD of the results obtained by NWCM, LCM, VAM, IAM, and TPMFP 
According to Table 8 and Figures 1 and 2, TPMFP is the best IFS, almost identical to the optimal solution (OS) in every case, obtained by the MODI method for TPs, and better than the solution obtained by the other existing methods such as NWCM, LCM, VAM, and IAM. The proposed TPMFP achieves more efficient IFS for a diverse set of TPs in fewer iterations.

The next section gives execution relationships for the distinctive eminent methods-NWCR, LCM, VAM, and the new method-and how to find the best result from the solutions obtained from disparate problems. Comparable assessments are performed and illustrated in the immediately following sections. The detailed representation of the numerical information in the table is given in Appendix B.

Table 9. A comparative results obtained by NWCM, LCM, VAM, IAM and New method for the FIVE balance transportation problem

\begin{tabular}{lllllllllllll}
\hline $\begin{array}{l}\text { Problem } \\
\text { chosen [2] }\end{array}$ & \multicolumn{3}{c}{ TCIFS $\left(\boldsymbol{I}_{\boldsymbol{F} S}\right)$} & \multicolumn{4}{c}{$\boldsymbol{4}$} & \multicolumn{4}{c}{$\begin{array}{c}\text { Percentage of Deviation from optimal } \\
\text { solution }\end{array}$} \\
\hline & NWCM & LCM & VAM & IAM & TPMFP & Optimal & NWCM & LCM & VAM & IAM & TPMFP \\
BTP.1 & 1,500 & 1,450 & 1,500 & 1,390 & 1,390 & 1,390 & 0.08 & 0.04 & 0.08 & 0.00 & 0.00 \\
BTP.2 & 226 & 156 & 156 & 156 & 156 & 156 & 44.9 & 0.00 & 0.00 & 0.00 & 0.00 \\
BTP.3 & 234 & 191 & 187 & 186 & 183 & 183 & 27.9 & 4.37 & 2.18 & 1.64 & 0.00 \\
BTP.4 & 4,285 & 2,455 & 2,310 & 2,365 & 2,330 & 2,170 & 97.5 & 13.13 & 6.45 & 8.98 & 7.37 \\
BTP.5 & 3,180 & 2,080 & 1,930 & 1,900 & 1,900 & 1,900 & 67.36 & 9.47 & 1.57 & 0.00 & 0.00 \\
\hline
\end{tabular}

As shown in Table 9, the proposed TPMFP method achieves a more promising IFS than the traditional algorithms considered in this study, as shown in Figure 3 and 4. Table 6 is depicted in Figure 3 as a bar graph and in Figure 4 as a line graph to demonstrate the significance of the proposed method, NEWA, in terms of transportation cost and IFS and PD, respectively:

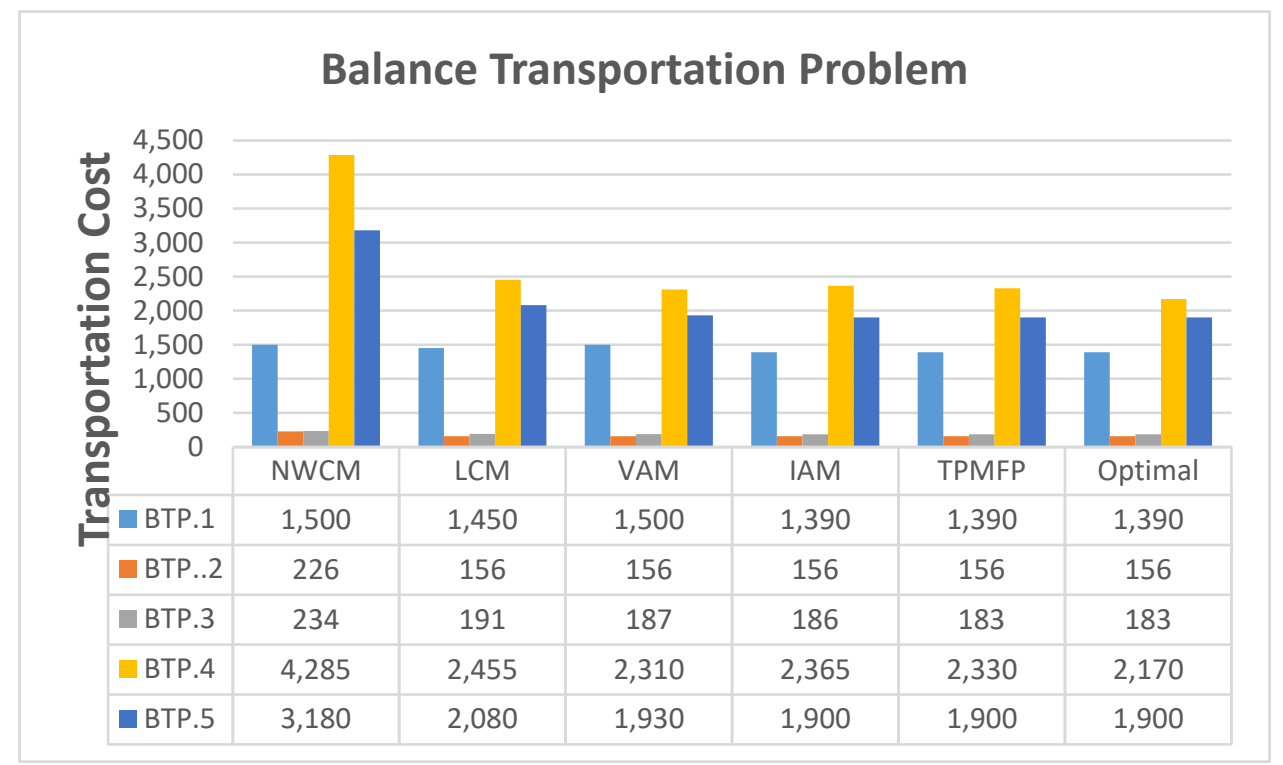

Figure 3. Comparative study of the result obtained by NWCM, LCM, VAM, IAM, and TPMFP 


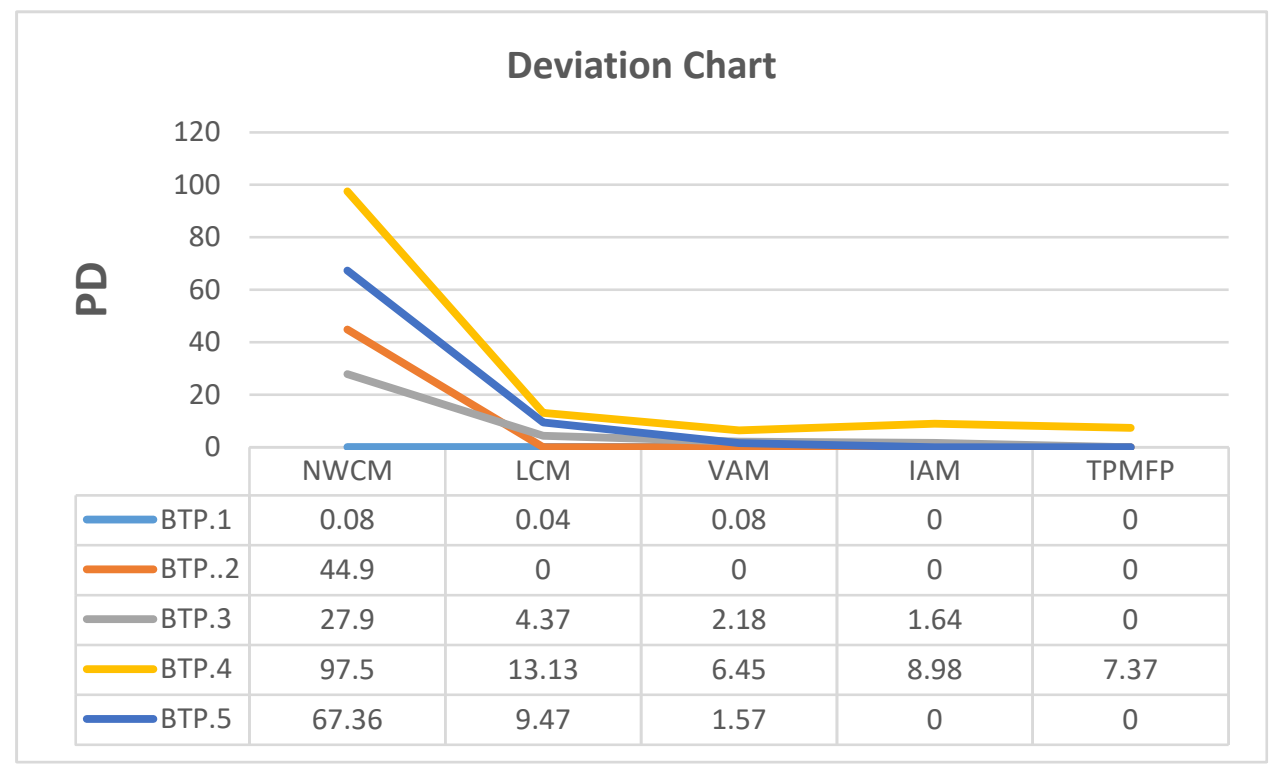

Figure 4. PD of the results obtained by NWCM, LCM, VAM, IAM, and TPMFP

According to Table 9, Figures 3 and 4, TPMFP is the best IFS, almost identical to the OS in all but one case in reaching the IFS, obtained by the MODI method for TPs, and better than the solution obtained by the other existing methods such as NWCM, LCM, VAM, and IAM. The proposed TPMFP achieves more efficient IFS for a diverse set of TPs in fewer iterations.

This section also compares the performance of various well-known methods (NWC, LCM, VAM, IAM, and TPMFP) developed using maximization TPs. The sections that follow perform and illustrate comparative assessments. Table 10 contains a detailed representation of the numerical data. Appendix $\mathbf{C}$ includes detailed data representations of the following five problems:

Table 10. Performance measure NEWA over NWCM, LCM, VAM, and IAM

\begin{tabular}{lllllllllllll}
\hline $\begin{array}{l}\text { Problem } \\
\text { chosen from }\end{array}$ & \multicolumn{3}{c}{} & \multicolumn{3}{c}{ TCIFS $\left(I_{\boldsymbol{F} S}\right)$} & & \multicolumn{3}{c}{$\begin{array}{l}\text { Optimal } \\
\text { Result }\left(\boldsymbol{O}_{\boldsymbol{c}}\right)\end{array}$} & $\begin{array}{l}\text { Percentage } \\
\text { solution }\end{array}$ \\
\hline Ahmed et al & NWCM & LCM & VAM & IAM & TPMFP & & NWCM & LCM & VAM & IAM & TPMFP \\
MTP-1. & 137 & 232 & 232 & 232 & 232 & 232 & -40.9 & 0.00 & 0.00 & 0.00 & 0.00 & friation \\
MTP-2. & 468 & 654 & 662 & 662 & 662 & 662 & -29.30 & -1.20 & 0.00 & 0.00 & 0.00 & optimal \\
MTP-3. & 5,570 & 8.020 & 8,000 & 8.020 & 8,020 & 8,020 & -30.5 & 0.00 & -0.24 & 0.00 & 0.00 & \\
MTP-4. & 36,795 & 46,760 & 46,760 & 46,700 & 46.700 & 46,760 & -21.31 & 0.00 & 0.00 & -0.12 & -0.12 \\
MTP-5. & 28,150 & 33,800 & 34,050 & 34,050 & 34,050 & 34,050 & -17.32 & -0.73 & 0.00 & 0.00 & 0.00 &
\end{tabular}

The TPMFP method's efficiency has also been tested by solving five benchmark problems and comparing performance across various well-known methods (NWCM, LCM, VAM, and IAM) in Table 10, where it is discovered that the TPMFP method yields a comparatively better result in all but one, which is not a better result. It can also be argued that the TPMFP may provide an outstanding IFS by requiring a minimum number of iterations. Table 10 also compares the bar graphs in Figure 5 and the line graphs in Figure 6 required to achieve the IFS for the proposed TPMFP algorithm. The comparative results shown in Table 10 are also represented using bar graphs and line graphs, as shown in Figures 5 and 6: 


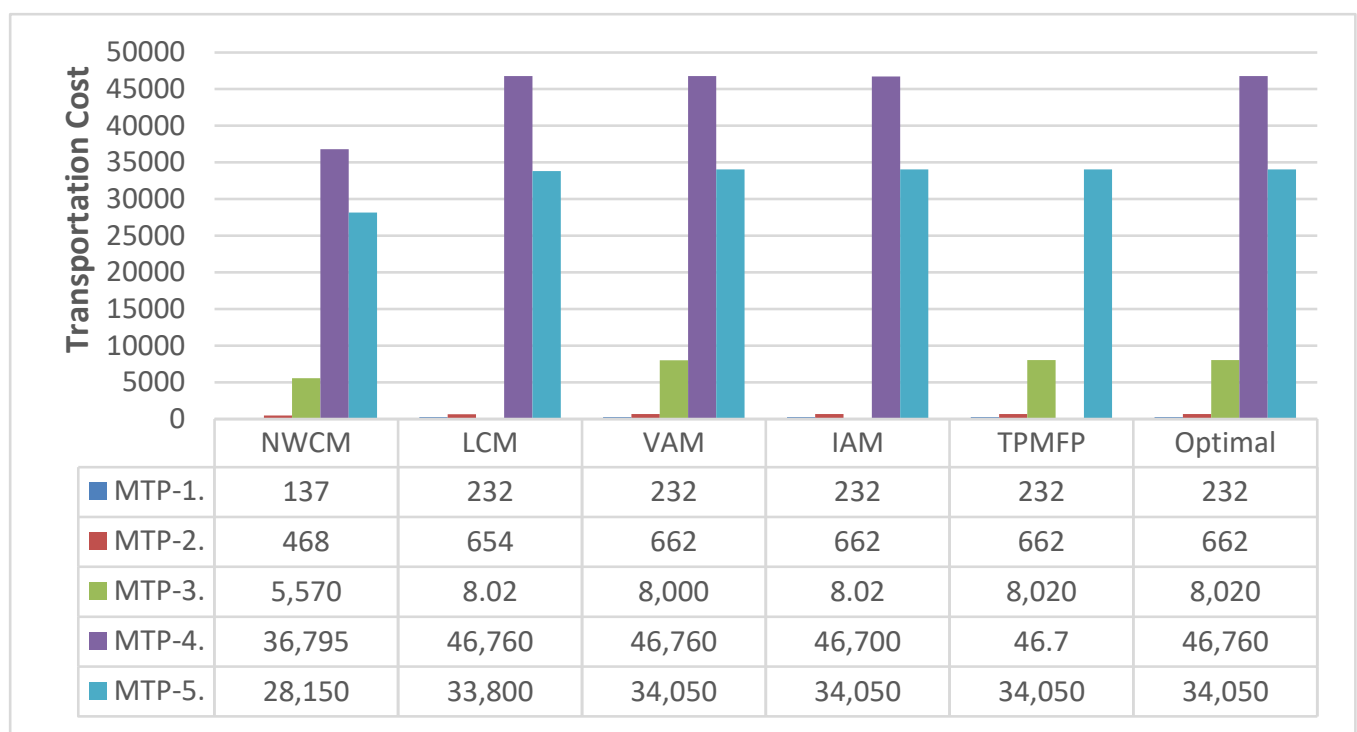

Figure 5. Comparative study of the result obtained by NWCM, CM, VAM, IAM, and TPMFP

Line graphs to indicate PD of the NWCM, LCM, VAM, and IAM with TPMFP from the minimal total cost solution obtained in Table 10 are presented in Figure 6:

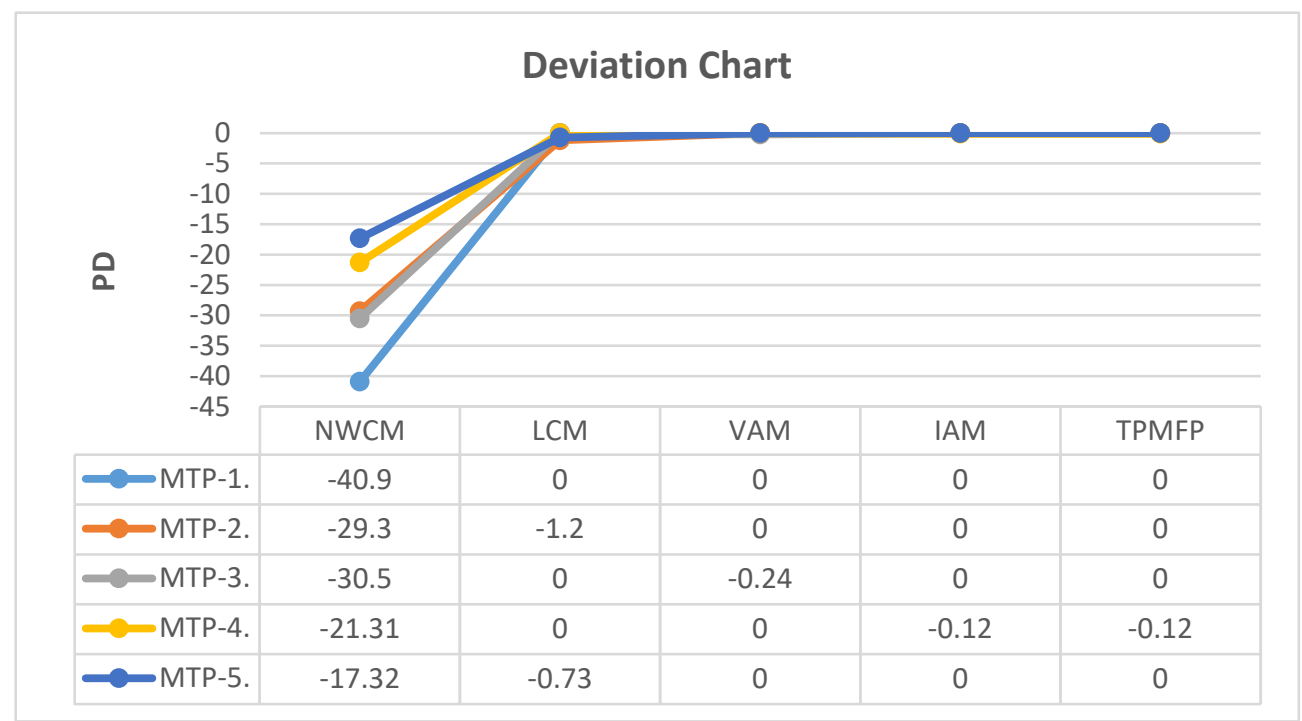

Figure 6. PD of the results obtained by NWCM, LCM, VAM, IAM, and TPMFP

Table 10 and Figures 5 and 6 demonstrate that the TPMFP method is more efficient than formalized inspection methods in determining IFS for the TP (NWCM, LCM, VAM, and IAM).

Comparative results acquired by NWCM, LCM, VAM, EDM and Ah. et al. and the proposed method for the ten benchmark instances are shown in the following Table 11. Detailed data information portrayal of these ten problems is provided in Appendix D: 
Table 11. Comparative results of NWCM, LCM, VAM, EDM, Ah.et.al, and TPMFP for 10 benchmark instances

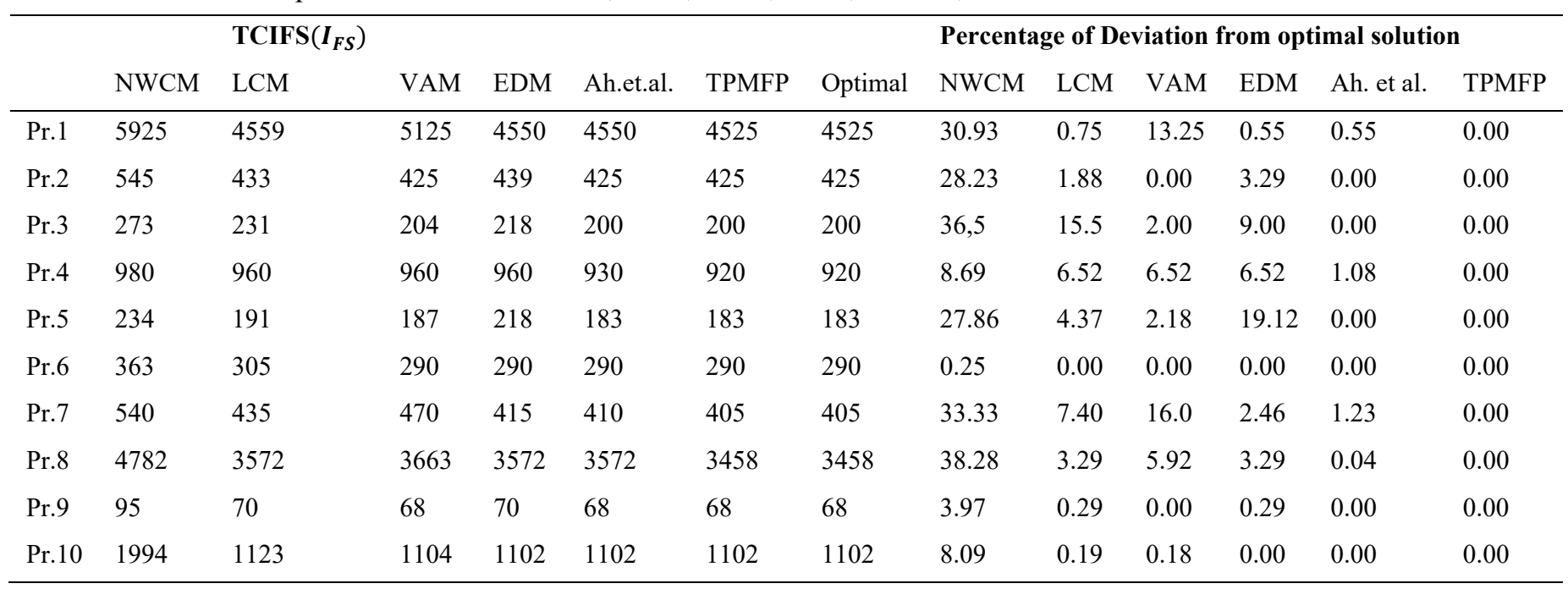

To compare the effectiveness of the TPMFP to existing methods, 10 different TPs were benchmarked and solved using NWCM, LCM, and VAM, EDM, Ah.et.al, and TPMFP with the results shown in Table 11. TPMFP clearly achieved the best OS in the fewest number of iterations for all problems. The comparative data from Table 11 is also depicted using bar graphs and line graphs, as shown in Figures 7 and 8 .

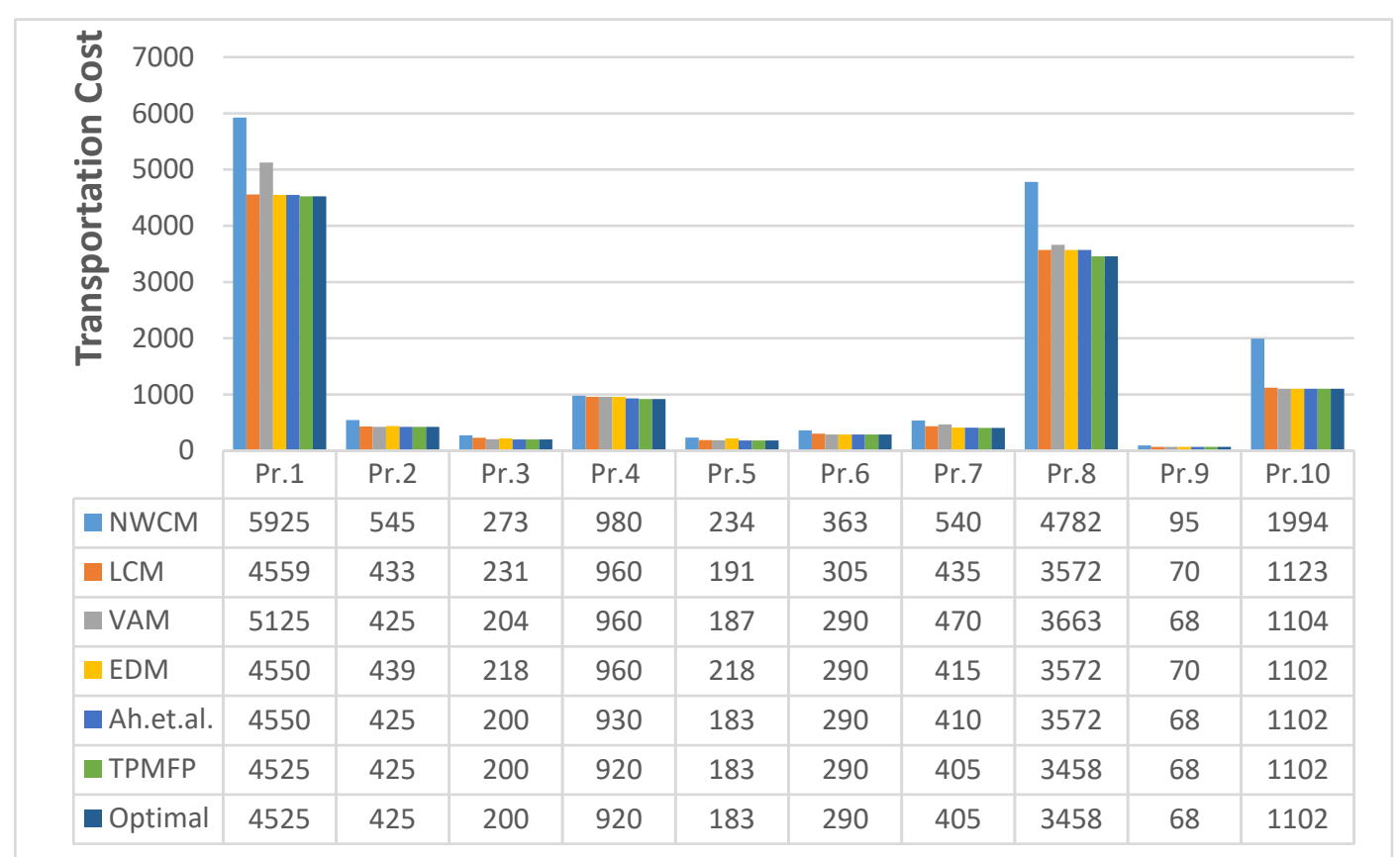

Figure 7. Comparative Stud of the Result obtained by NWCM, LCM, VAM, EDM, Ah. et al., and TPMFP 


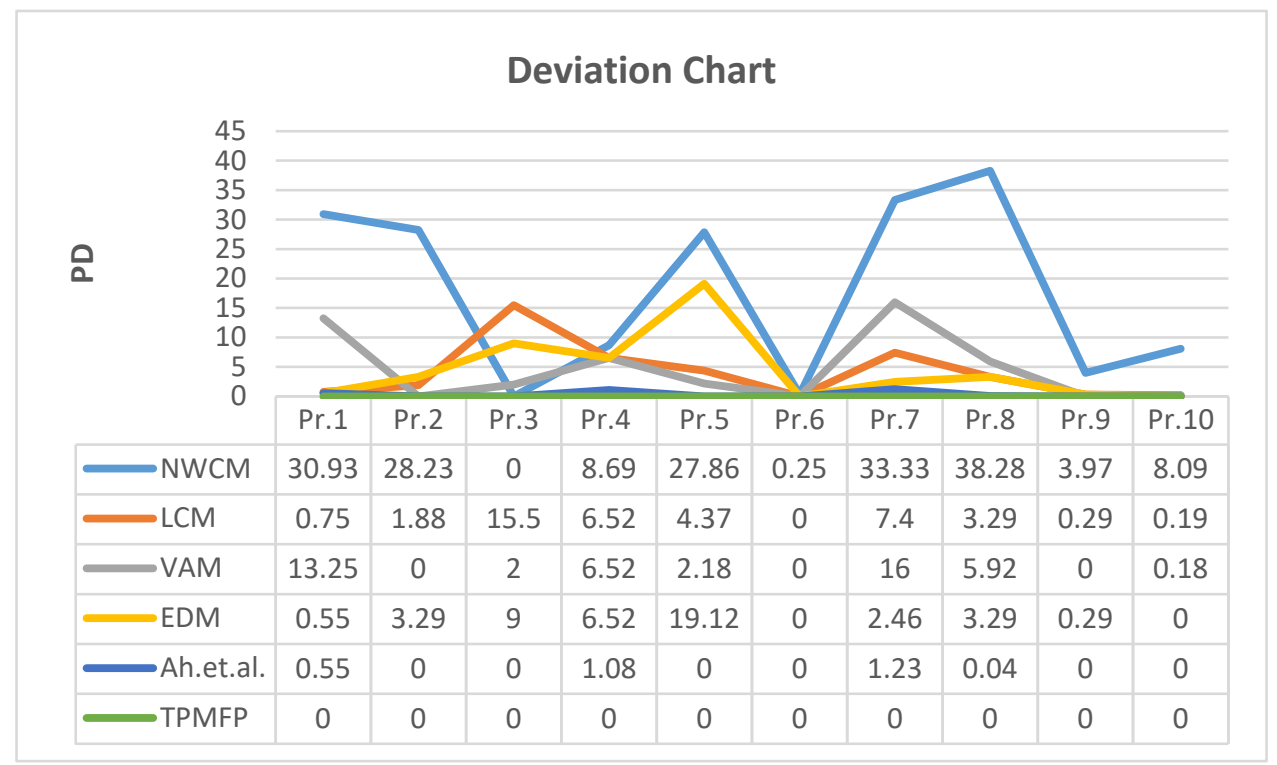

Figure 8. PD of the results obtained by NWCM, LCM, VAM, EDM, Ah. et al, and TPMFP

As evidenced by the above results in Table 11, Figure 7, and Figure 8, the proposed method outperformed NWCM, LCM, VAM EDM, Ah. et al. for all seven TPs listed in Table 11.

This section also compares the performance of various well-known methods (NWC, LCM, VAM, EDM, Ah. et al., and PROPOSED METHOD) as well as the solutions obtained from disparate problems. In the sections that follow, comparative assessments are performed and illustrated. Appendix $\mathbf{E}$ contains a detailed representation of the numerical data.

Table 12. Comparative results of NWCM, LCM, VAM, EDM, Ah. et al., and TPMFP for 10 benchmark instances

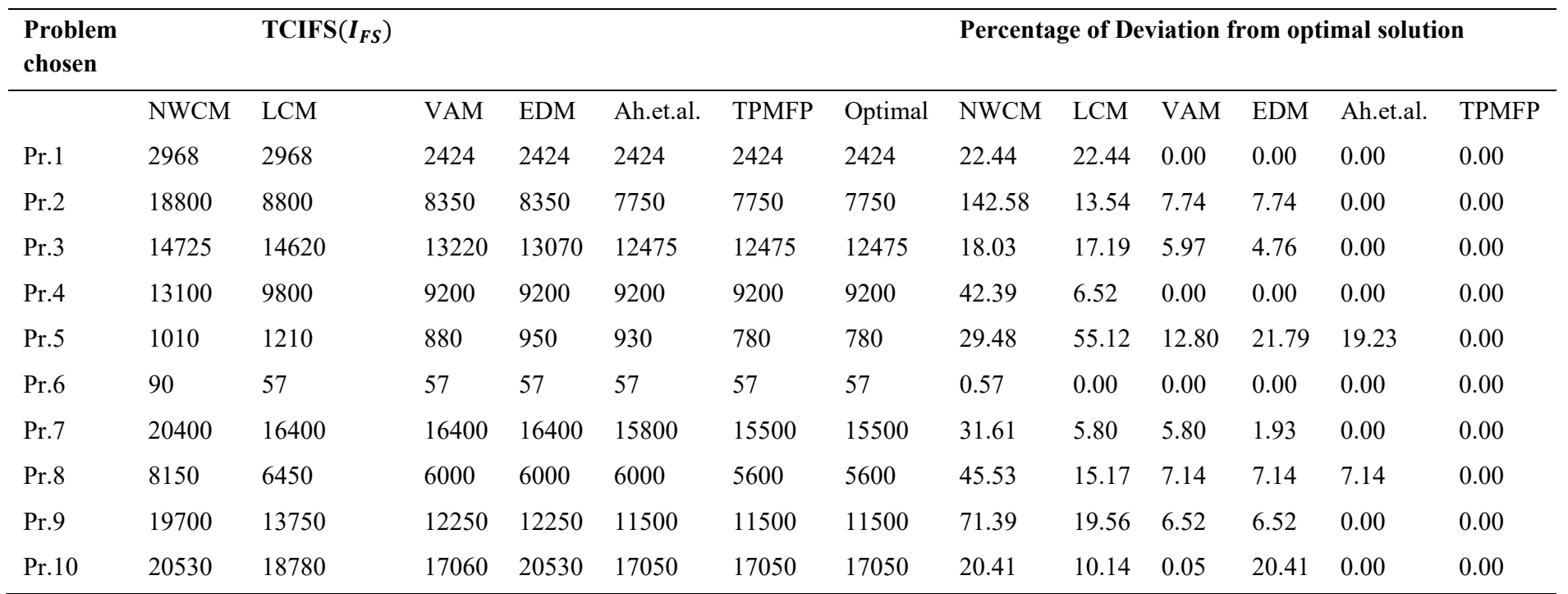

To compare the TPMFP's effectiveness to existing methods, 10 different TPs were benchmarked and solved using NWCM, LCM, and VAM, EDM, Ah. et. al., and TPMFP, with the results shown in Table 12.TPMFP clearly achieved the best IFS with the fewest iterations for all problems. Figures 9 and 10 use bar graphs and line graphs to illustrate the results graphically. 


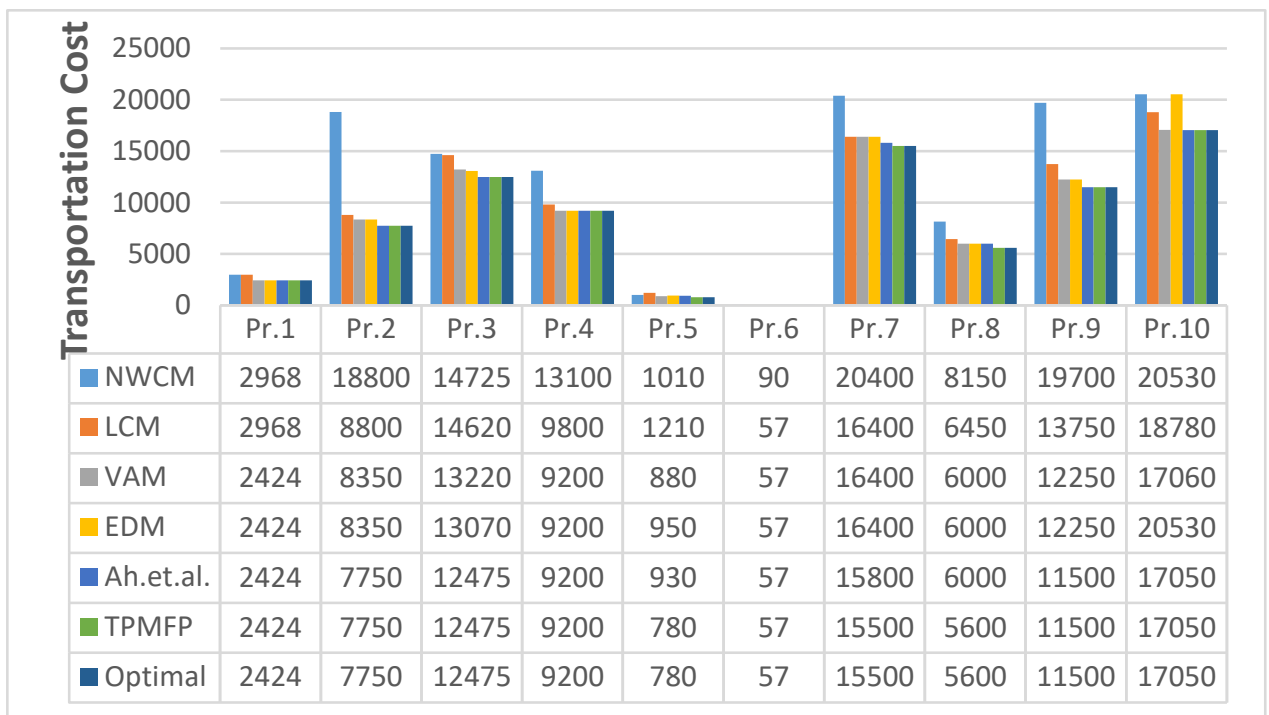

Figure 9. A comparison of the results obtained by the NWCM, LCM, VAM, EDM, Ah. et al., and TPMFP

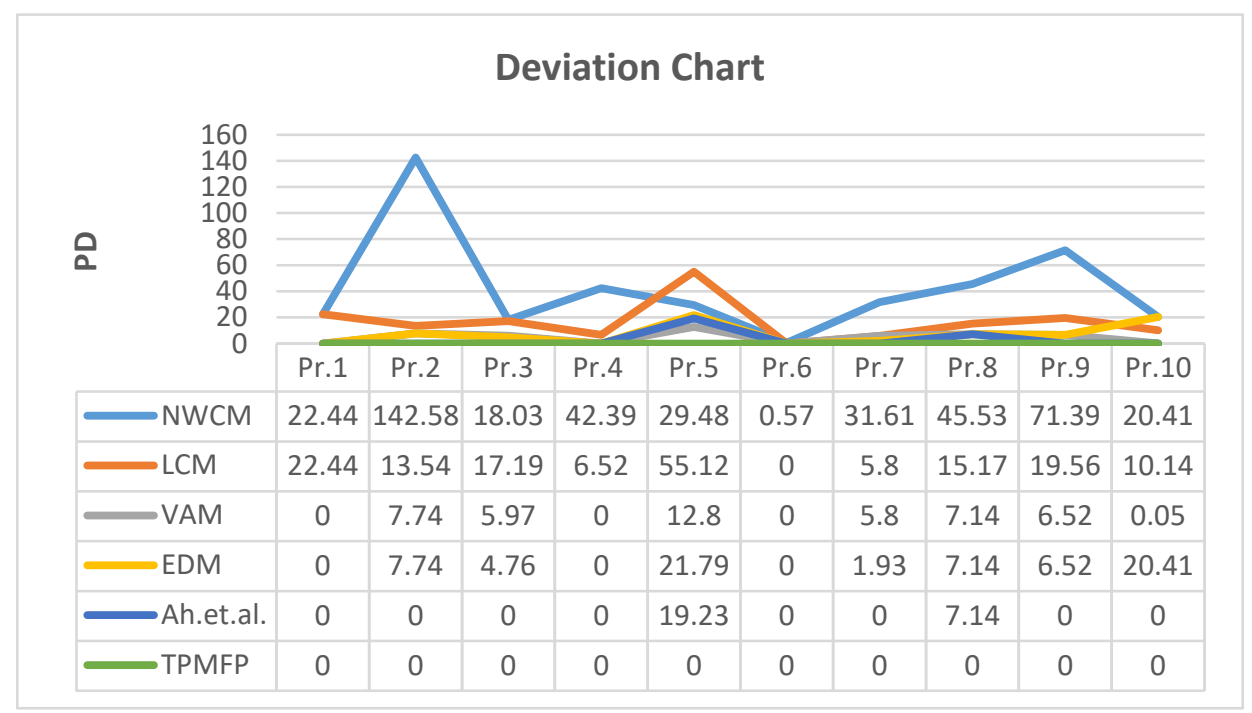

Figure 10. PD of the NWCM, LCM, VAM, EDM, Ah. et al., and TPMFP results

Tables 12, Figures 9, and 10 show that the TPMFP method is more efficient than formalized inspection methods in determining OS for the TP (NWCM, LCM, VAM, EDM, and Ah. et al.). Let us consider the following TP to find out the minimum transportation cost.

\subsection{Numerical Illustration (TP)}

A numerical example has been developed for resolving the transportation problem using the proposed algorithm, which is provided below.

Table 13. Let us consider the following TP to find out the minimum transportation cost

\begin{tabular}{lllll}
\hline & $\boldsymbol{S}_{\mathbf{1}}$ & $\boldsymbol{S}_{\mathbf{2}}$ & $\boldsymbol{S}_{\mathbf{3}}$ & \\
\hline $\boldsymbol{D}_{\mathbf{1}}$ & 6 & 8 & 10 & 150 \\
$\boldsymbol{D}_{\mathbf{2}}$ & 7 & 11 & 11 & 175 \\
$\boldsymbol{D}_{\mathbf{3}}$ & 4 & 5 & 12 & 275 \\
& 100 & 300 & 200 & \\
\hline
\end{tabular}


Table 14. Construct a Predecessor Transportation Cost Table (PTCT) from the given transportation problem

\begin{tabular}{llllllll}
\hline & $\boldsymbol{S}_{\mathbf{1}}$ & $\boldsymbol{S}_{\mathbf{2}}$ & $\boldsymbol{S}_{\mathbf{3}}$ & $\boldsymbol{D}_{\mathbf{1}}$ & $\boldsymbol{D}_{\mathbf{2}}$ & $\boldsymbol{D}_{\mathbf{3}}$ & \\
\hline $\boldsymbol{D}_{\mathbf{1}}$ & 6 & 8 & 10 & 0 & $\infty$ & $\infty$ & 150 \\
$\boldsymbol{D}_{\mathbf{2}}$ & 7 & 11 & 11 & $\infty$ & 0 & $\infty$ & 175 \\
$\boldsymbol{D}_{\mathbf{3}}$ & 4 & 5 & 12 & $\infty$ & $\infty$ & 0 & 275 \\
$\boldsymbol{S}_{\mathbf{1}}$ & 0 & $\infty$ & $\infty$ & 6 & 8 & 10 & 200 \\
$\boldsymbol{S}_{\mathbf{2}}$ & $\infty$ & 0 & $\infty$ & 7 & 11 & 11 & 100 \\
$\boldsymbol{S}_{\mathbf{3}}$ & $\infty$ & $\infty$ & 0 & 4 & 5 & 12 & 300 \\
& 200 & 100 & 300 & 150 & 175 & 275 & \\
\hline
\end{tabular}

Calculate $\frac{\sum_{i=1}^{m} a_{i}}{m}=\frac{\sum_{j=1}^{n} b_{j}}{m}=\overline{\frac{600}{5}}=120=\varnothing$ and $\emptyset \cong 100$

Table 15. Distinguish the source or Demand cell close $\varnothing$

\begin{tabular}{llllllll}
\hline & $\boldsymbol{S}_{\mathbf{1}}$ & $\boldsymbol{S}_{\mathbf{2}}$ & $\boldsymbol{S}_{\mathbf{3}}$ & $\boldsymbol{D}_{\mathbf{1}}$ & $\boldsymbol{D}_{\mathbf{2}}$ & $\boldsymbol{D}_{\mathbf{3}}$ & \\
\hline $\boldsymbol{D}_{\mathbf{1}}$ & 6 & 8 & 10 & 0 & $\infty$ & $\infty$ & 150 \\
$\boldsymbol{D}_{\mathbf{2}}$ & 7 & 11 & 11 & $\infty$ & 0 & $\infty$ & 175 \\
$\boldsymbol{D}_{\mathbf{3}}$ & 4 & $5^{*} 100$ & 12 & $\infty$ & $\infty$ & 0 & 175 \\
$\boldsymbol{S}_{\mathbf{1}}$ & 0 & $\infty$ & $\infty$ & 6 & 7 & 4 & 200 \\
$\boldsymbol{S}_{\mathbf{2}}$ & $\infty$ & 0 & $\infty$ & 8 & 11 & 5 & 100 \\
$\boldsymbol{S}_{\mathbf{3}}$ & $\infty$ & $\infty$ & 0 & 10 & 11 & 12 & 300 \\
& 200 & 0 & 300 & 150 & 175 & 275 &
\end{tabular}

Table 16. Next utilizing the above Algorithm and goes to head

\begin{tabular}{llllllll}
\hline & $\boldsymbol{S}_{\mathbf{1}}$ & $\boldsymbol{S}_{\mathbf{2}}$ & $\boldsymbol{S}_{\mathbf{3}}$ & $\boldsymbol{D}_{\mathbf{1}}$ & $\boldsymbol{D}_{\mathbf{2}}$ & $\boldsymbol{D}_{\mathbf{3}}$ & \\
\hline $\boldsymbol{D}_{\mathbf{1}}$ & 6 & 8 & 10 & 0 & $\infty$ & $\infty$ & 150 \\
$\boldsymbol{D}_{\mathbf{2}}$ & 7 & 14 & 11 & $\infty$ & 0 & $\infty$ & 175 \\
$\boldsymbol{D}_{\mathbf{3}}$ & 4 & 0 & 12 & $\infty$ & $\infty$ & $\theta$ & 175 \\
$\boldsymbol{S}_{\mathbf{1}}$ & 0 & $\infty$ & $\infty$ & 6 & 7 & $4^{*} 175$ & 25 \\
$\boldsymbol{S}_{\mathbf{2}}$ & $\infty$ & $\theta$ & $\infty$ & 8 & 41 & $5^{*} 100$ & 0 \\
$\boldsymbol{S}_{\mathbf{3}}$ & $\infty$ & $\infty$ & 0 & 10 & 11 & 12 & 300 \\
& 200 & 0 & 300 & 150 & 175 & 0 & \\
\hline
\end{tabular}

Table 17. Next utilizing the above Algorithm and goes to head

\begin{tabular}{llllllll}
\hline & $\boldsymbol{S}_{\mathbf{1}}$ & $\boldsymbol{S}_{\mathbf{2}}$ & $\boldsymbol{S}_{\mathbf{3}}$ & $\boldsymbol{D}_{\mathbf{1}}$ & $\boldsymbol{D}_{\mathbf{2}}$ & $\boldsymbol{D}_{\mathbf{3}}$ & \\
\hline $\boldsymbol{D}_{\mathbf{1}}$ & $6 * 25$ & 8 & 10 & 0 & $\infty$ & $\infty$ & 125 \\
$\boldsymbol{D}_{\mathbf{2}}$ & 7 & 11 & 11 & $\infty$ & 0 & $\infty$ & 175 \\
$\boldsymbol{D}_{\mathbf{3}}$ & 0 & 0 & 12 & $\infty$ & $\infty$ & $\theta$ & 0 \\
$\boldsymbol{S}_{\mathbf{1}}$ & 0 & $\infty$ & $\infty$ & 6 & 7 & $4 * 175$ & 25 \\
$\boldsymbol{S}_{\mathbf{2}}$ & $\infty$ & $\theta$ & $\infty$ & 8 & 11 & $5 * 100$ & 0 \\
$\boldsymbol{S}_{\mathbf{3}}$ & $\infty$ & $\infty$ & 0 & 10 & 11 & 12 & 300 \\
& 0 & 0 & 300 & 150 & 175 & 0 &
\end{tabular}

Table 18. Next utilizing the above Algorithm and goes to head

\begin{tabular}{llllllll}
\hline & $\boldsymbol{S}_{\mathbf{1}}$ & $\boldsymbol{S}_{\mathbf{2}}$ & $\boldsymbol{S}_{\mathbf{3}}$ & $\boldsymbol{D}_{\mathbf{1}}$ & $\boldsymbol{D}_{\mathbf{2}}$ & \multicolumn{1}{c}{$\boldsymbol{D}_{\mathbf{3}}$} & \\
\hline $\boldsymbol{D}_{\mathbf{1}}$ & 0 & 8 & 10 & 0 & $\infty$ & $\infty$ & 125 \\
$\boldsymbol{D}_{\mathbf{2}}$ & 7 & 11 & 11 & $\infty$ & 0 & $\infty$ & 175 \\
$\boldsymbol{D}_{\mathbf{3}}$ & 0 & 0 & 12 & $\infty$ & $\infty$ & $\theta$ & 0 \\
$\boldsymbol{S}_{\mathbf{1}}$ & 0 & $\infty$ & $\infty$ & $6 * 25$ & 7 & $4 * 175$ & 0 \\
$\boldsymbol{S}_{\mathbf{2}}$ & $\infty$ & 0 & $\infty$ & 8 & 11 & $5^{*} 100$ & 0 \\
$\boldsymbol{S}_{\mathbf{3}}$ & $\infty$ & $\infty$ & 0 & $10 * 125$ & 11 & 12 & 175 \\
& 0 & 0 & 300 & 0 & 175 & 0 & \\
\hline
\end{tabular}


Table 19. Next utilizing the above Algorithm and goes to head

\begin{tabular}{llllllll}
\hline & $\boldsymbol{S}_{\mathbf{1}}$ & $\boldsymbol{S}_{\mathbf{2}}$ & \multicolumn{1}{c}{$\boldsymbol{S}_{\mathbf{3}}$} & $\boldsymbol{D}_{\mathbf{1}}$ & $\boldsymbol{D}_{\mathbf{2}}$ & $\boldsymbol{D}_{\mathbf{3}}$ & \\
\hline $\boldsymbol{D}_{\mathbf{1}}$ & 0 & 8 & 0 & 0 & $\infty$ & $\infty$ & 0 \\
$\boldsymbol{D}_{\mathbf{2}}$ & 7 & 11 & $11^{*} 175$ & $\infty$ & 0 & $\infty$ & 0 \\
$\boldsymbol{D}_{\mathbf{3}}$ & 0 & 0 & 12 & $\infty$ & $\infty$ & $\theta$ & 0 \\
$\boldsymbol{S}_{\mathbf{1}}$ & 0 & $\infty$ & $\infty$ & $6 * 25$ & 7 & $4 * 175$ & 0 \\
$\boldsymbol{S}_{2}$ & $\infty$ & 0 & $\infty$ & 8 & 11 & $5 * 100$ & 0 \\
$\boldsymbol{S}_{\mathbf{3}}$ & $\infty$ & $\infty$ & 0 & $10 * 125$ & 11 & 12 & 175 \\
& 0 & 0 & 0 & 0 & 175 & 0 &
\end{tabular}

Table 20. Next utilizing the above Algorithm and goes to head

\begin{tabular}{llllcccc}
\hline & $\boldsymbol{S}_{\mathbf{1}}$ & $\boldsymbol{S}_{\mathbf{2}}$ & $\boldsymbol{S}_{\mathbf{3}}$ & $\boldsymbol{D}_{\mathbf{1}}$ & $\boldsymbol{D}_{\mathbf{2}}$ & $\boldsymbol{D}_{\mathbf{3}}$ & \\
\hline $\boldsymbol{D}_{\mathbf{1}}$ & 0 & 8 & 0 & 0 & $\infty$ & $\infty$ & 0 \\
$\boldsymbol{D}_{\mathbf{2}}$ & 7 & 14 & 0 & $\infty$ & 0 & $\infty$ & 0 \\
$\boldsymbol{D}_{\mathbf{3}}$ & 0 & 0 & 12 & $\infty$ & $\infty$ & $\theta$ & 0 \\
$\boldsymbol{S}_{\mathbf{1}}$ & 0 & $\infty$ & $\infty$ & $6 * 25$ & 7 & $4 * 175$ & 0 \\
$\boldsymbol{S}_{\mathbf{2}}$ & $\infty$ & $\theta$ & $\infty$ & 8 & 11 & $5 * 100$ & 0 \\
$\boldsymbol{S}_{\mathbf{3}}$ & $\infty$ & $\infty$ & 0 & $10 * 125$ & $11^{*} 175$ & 12 & 0 \\
& 0 & 0 & 0 & 0 & 0 & 0 & \\
\hline
\end{tabular}

Transportation cost $=6 \times 25+4 \times 175+5 \times 100+10 \times 125+11 \times 175=4,525$

\section{Conclusion}

Any mathematical algorithm's modification is a never-ending process to achieve the best possible result. This paper presents some new strategies for taking care of the maximal network flow problem with applications to solving the transportation problem. By means of this, we expect to propose an algorithm that will realize maximum flow in a network flow problem, including a lower number of augmentations. For this, we considered different kinds of algorithms, for example, Ford-Fulkerson, Edmonds-Karp, Md. Al-Amin Khan, Faruque Ahmed, Chintan, and Deepak Garg, modified Edmonds-Karp algorithms. This proposed algorithm requires fewer iterations and augmentations to obtain maximum flow in a network flow problem more precisely in comparison with the other prominent algorithms. In addition, Transportation of product dissemination from several original points to several destinations minimizes the cost of transportation. Numerous researchers have focused on solving this problem by utilizing various approaches. The Northwest, Least Cost, and Vogel's Approximation methods are the most noticeable and renowned for finding an initial feasible solution for a TP out of all the existing methods and techniques in the writing. The Modified Distribution (MODI) Method and the Stepping Stone Method are the most acceptable methods for finding the minimal total cost solution to the transportation problem. These well-known techniques for minimizing total cost begin with an Initial Feasible Solution (IFS). In this way, an IFS acts as the foundation of an optimal cost solution technique for any TP. The better the IFS, the fewer iterations it takes to reach the optimal solution. However, in this research paper, we discuss a new alternative method, a modified max flow algorithm, which often gives an optimal solution to the transportation problem.

In this research paper, we first examine different initial solutions, giving methods to achieve initial, feasible solutions to balanced and unbalanced transportation problems. The TPMFP is extremely straightforward, straightforward, and simple to actualize. This strategy requires a minimum number of steps to achieve optimality as compared to the existing methods. The comparative assessment shows that both the new method and existing methods are proficient when compared with the considered approaches of this paper in terms of the quality of the solution. However, in practice, when researchers and practitioners deal with large-sized transportation problems, since the existing well-known exact optimal cost solution method (SSM) deals fully with the path tracing technique, it becomes very difficult to solve large-scale transportation problems. Subsequently, we intend to devote ourselves in the near future to proposing an alternate optimal approach that gets rid of this difficulty.

\section{Acknowledgments}

The authors would like to express their gratitude to Prof. Ranjith Edirisinghe, Dean of the Faculty of Applied Sciences, and Dr. Harshani Wijewardane, Head of the Department of Physical Sciences, Faculty of Applied Sciences, Rajarata University of Sri Lanka, Mihintale, Sri Lanka, for their invaluable assistance and the valuable time they provided to make this investigation a success. 


\section{References}

Ahmed, M. M., Khan, A. R., Ahmed, F., \& Uddin, M. S. (2016). Incessant Allocation Method for Solving Transportation Problems. American Journal of Operations Research, 6(3), 236-244. https://doi.org/10.4236/ajor.2016.63024

Ahmed, M. M., Khan, A. R., Ahmed, F., \& Uddin, M. S. (2017). An Innovative Approach to Obtain an Initial Basic Feasible Solution for the Transportation Problems. Journal of Physical Sciences, 22, 23-42.

Ahmed, M. M., Khan, A. R., Uddin, M. S., \& Ahmed, F. (2016). A New Approach to Solve Transportation Problems. Open Journal of Optimization, 5(1), 22-30. https://doi.org/10.4236/ojop.2016.51003

Ahuja, R. K., \& Orlin, J. B. (1989). A fast and simple algorithm for the maximum flow problem. Operations Research, 37(5), 748-759. https://doi.org/10.1287/opre.37.5.748

Altner, D., Ergun, O., \& Uhan, N. (2010). The maximum flow network interdiction problem: Valid inequalities, integrality gaps, and approximability. Operations Research Letters, 38(1), 33-38. https://doi.org/10.1016/j.orl.2009.09.013

Babu, M. A., Helal, M. A., Hasan, M. S., \& Das, U. K. (2013). Lowest Allocation Method (LAM): A New Approach to Obtain Feasible Solution of Transportation Model. International Journal of Scientific and Engineering Research, 4(11), 1344-1348.

Chandler Burfield. (2013). Floyd-Warshall Algorithm. Retrieved from https://studylib.net/doc/10622823/floyd-warshall-algorithm-chandler-burfield-february-20--2

Charnes, A., Cooper, W. W., \& Henderson, A. (1953). An introduction to Linear Programming. Wiley, New Work.

Christofides, N. (2016). Graph Theory: An Algorithmic Approach. Academic Press, New York, London, San Francisco.

Dash, P., Rahman, M. M., \& Akter, M. S. (2019). Developing Algorithm to Obtain the Maximum Flow in a Network Flow Problem. Journal of Advanced Research in Dynamical and Control Systems, 11(2), 455-459.

Deshmukh, N. M. (2012). An Innovative Method for Solving Transportation Problem. International Journal of Physics and Mathematical Sciences, 2(3), 86-91.

Dimitri, P. Bertsekas. (1998). Network Optimization: Continuous and Discrete Models. Publisher's Cataloging-in-Publication Data.

Douglas, S. (2010). Altner The Maximum Flow Network Interdiction Problem: Valid Inequalities, Integrality Gaps, and Approximability". Operations Research Letters, 38(1), 33-38.

E. A. Dinic. (1970). Algorithm for Solution of a Problem of Maximum Flow in Networks with Power Estimation. Soviet Mathematics Doklady, 11, 1277-1280.

Edmonds, J., \& Karp, R. M. (1972). Theoretical Improvements in Algorithmic Efficiency for Network Flow Problems. Journal of the ACM, 19(2), 248-264. https://doi.org/10.1145/321694.321699

Ekanayake, E. M. U. S. B., Daundasekara, W. B., \& Perera, S. P. C. (2021). Solution of a Transportation Problem using Bipartite Graph. Global Journal of Science Frontier Research: F Mathematics and Decision Sciences, 21(1).

Ekanayake, E. M. U. S. B., Perera, S. P. C., Daundasekara, W. B., \& Juman Z. A. M. S. (2021). An Effective Alternative New Approach in Solving, Transportation Problems. American Journal of Electrical and Computer Engineering, 5(1), 1-8. https://doi.org/10.11648/j.ajece.20210501.11

Ekanayake, E. M. U. S. B., Perera, S. P. C., Daundasekara, W. B., \& Juman, Z. A. M. S. (2020). A Modified Ant Colony Optimization Algorithm for Solving a Transportation Problem. Journal of Advances in Mathematics and Computer Science, 35(5), 83-101. https://doi.org/10.9734/jamcs/2020/v35i530284

Elias, P., Feinstein. A., \& Shannon, C. E. (1956). A note on the maximum flow through a network. IRE Transactions on Information Theory, 2(4), 117-119. https://doi.org/10.1109/TIT.1956.1056816

Fabian Latorre. (2012). The Maxflow problem and a generalization to simplicial complexes.

Ford, L. R., \& Fulkerson, D. R. (1956). Maximal Flow through a Network. Canadian Journal of Mathematics, 8 , 399-404. https://doi.org/10.4153/CJM-1956-045-5

Ford, L. R., \& Fulkerson, D. R. (1962). Flows in Networks. Princeton University Press, Princeton. 
Fulkerson, D. R., \& Dantzig, G. B. (1955). Computation of maximum flow in network. Naval Research Logistics, 2(4), 277-283. https://doi.org/10.1002/nav.3800020407

Girmay, N., \& Sharma, T. (2013). Balance An Unbalanced Transportation Problem By A Heuristic approach. International Journal of Mathematics And Its Applications, 1(1), 13-19.

Goldberg, A. V., \& Tarjan, R. E. (1988). A new approach to the maximum-flow problem. Journal of the ACM, 35(4), 921-940. https://doi.org/10.1145/48014.61051

Harris, T. E., \& Ross, F. S. (1956). Fundamentals of a method for Evaluating rail net capacities. (U) RAND Corporation, Research Memorandum RM-1573.

Hitchcock, F. L. (1941). The distribution of a product from several sources to numerous localities. Journal of Mathematics and Physics, 20(1-4), 224-230. https://doi.org/10.1002/sapm1941201224

Hu, T. C. (1970). Integer Programming and Network Flows. Addison-Wesley Publishing Company, Ontario.

Karzanova, V. (1974). Determining the Maximal Row in a Network by the Method of Preflows. Soviet Math. Dokl., 15, 434-437.

Khan, A. R., Vilcu, A., Sultana, N., \& Ahmed, S. S. (2015). Determination of Initial Basic Feasible Solution of a Transportation Problem: A TOCM-SUM Approach.

Kulkarni, S. S., \& Datar, H. G. (2010). On Solution to Modified Unbalanced Transportation problem. Bulletin of the Marathwada Mathematical Society, 11(2), 20-26.

Mallick, K. K., Khan, A. R., Ahmed, M. M., Arefin, Md. S., \& Uddin, Md. S. (2016). Modified EDMONDS-KARP Algorithm to Solve Maximum Flow Problems. Open Journal of Applied Sciences, 6(2), 131-140. https://doi.org/10.4236/ojapps.2016.62014

Minieka, E. (1978). Optimization Algorithms for Networks and Graphs. Marcel Dekker, Inc., New York and Basel.

Pannerselvam, R. (2010). Operation Research (2nd ed.). PHI Learning Pvt. Ltd. New Delhi, India.

Prabha, S. K., \& Vimala, S. (2016). An modified method for solving balanced fuzzy transporation problem for maximizing the profit. International Journal of Pure and Applied Mathematics, 106(5), 45-52.

Rayand, G. G., \& Hosain, M. E. (2007). Operation Research.

Seethalakshmy, A., \& Srinivasan, N. (2019). Solving Real-Life Problem Using Transportation. International Journal of Innovative Technology and Exploring Engineering, 8(10), 4284-4287.

Sleator, D. D., \& Tarjan, R. E. (1983). A Data Structure for Dynamic Trees. Journal of Computer and System Sciences, 26(3), 362-391. https://doi.org/10.1016/0022-0000(83)90006-5

Swarup, K., Gupta, P. K., \& Mohan, M. (1955). Operation Research (7th ed.) (Reprint). Sultan Chand \& Sons. New Delhi, India.

Uddin, M. S., Khan, A. R., Kibria, C. G., \& Raeva, I. (2016). Improved Least Cost Method to Obtain a Better IBFS to the Transportation Problem. Journal of Applied Mathematics \& Bioinformatics, 6(2), 1-20.

Uthpala Ekanayake, Wasantha Daundasekara, \& Pantalian Perera. (2020). An Approach for Solving Minimum Spanning Tree Problem and Transportation Problem Using Modified Ant Colony Algorithm. North American Academic Research, 3(9), 28-45.

Zhipeng Jiang, Xiaodong Hu, \& Suixiang Gao. (2013). A Parallel Ford-Fulkerson Algorithm For Maximum Flow Problem. https://www.semanticscholar.org/paper/A-Parallel-Ford-Fulkerson-Algorithm-For-Maximum-Jiang-Hu/8e7 7c56a615f078d982223a61246b2570503b6ea 


\section{Appendix A}

Unbalanced Transportation Problems

\section{Problems Data of the problems}

UTP-1 $\quad c_{i j}=[61014 ; 121921 ; 151417], s_{i j}=[50,50,50], d_{i j}=[30,40,55]$

UTP-2 $\quad c_{i j}=[10843 ; 1214202 ; 692325], s_{i j}=[500,400,300], d_{i j}=[250,350,600,150]$

UTP-3 $\quad c_{i j}=[1210613 ; 1981625 ; 17151520 ; 23222612], s_{i j}=[150,200,600,225]$,

$d_{i j}=[300,500,75,100]$

UTP-4 $\quad c_{i j}=[58663 ; 47765 ; 84664], s_{i j}=[800,500,900], d_{i j}=[400,400,500,400,800]$

UTP-5 $\quad c_{i j}=[54865 ; 45432 ; 36584], s_{i j}=[600,400,1000], d_{i j}=[450,400,200,250,300]$

\section{Appendix B}

Balanced Transportation Problems

\section{Problems Data of the problems}

BTP-1 $\quad c_{i j}=[435 ; 654 ; 8107], s_{i j}=[90,80,100], d_{i j}=[70,120,80]$

BTP-2 $\quad c_{i j}=[4695 ; 2641 ; 5729], s_{i j}=[16,12,15], d_{i j}=[12,14,9,8]$

BTP-3 $\quad c_{i j}=[571053 ; 8691214 ; 10981015], s_{i j}=[5,10,10], d_{i j}=[3,3,10,5,4]$

$c_{i j}=$

BTP-4 [12 413189 2; 91610715 11; 491089 7; 931264 5; 7115182 7; 168451 10], $s_{i j}=[120,80,50,90,100,60], d_{i j}=[75,85,140,40,95,65]$

BTP-5 $c_{i j}=[127381066 ; 697128124 ; 101284993 ; 85116793 ; 76811956], s_{i j}=$ $[60,80,70,100,90], d_{i j}=[20,30,40,70,60,80,100]$

\section{Appendix C}

Profit maximization Transportation Problems

\begin{tabular}{ll}
\hline Problems & Data of the problems \\
\hline MTP-1 & $c_{i j}=[6415 ; 8927 ; 4362], s_{i j}=[14,18,7], d_{i j}=[6,10,15,8]$ \\
MTP-2 & $c_{i j}=[141975 ; 166129 ; 616520], s_{i j}=[10,12,18], d_{i j}=[9,14,7,10]$ \\
MTP-3 & $c_{i j}=[16141125 ; 18291227 ; 14231612], s_{i j}=[140,180,70], d_{i j}=[60,100,150,80]$ \\
& $c_{i j}=$ \\
MTP-4 & {$[352233162012 ; 142128301524 ; 551817292619 ; 211615173128 ; 452316112250], s_{i j}=$} \\
& {$[320,180,200,300,300], d_{i j}=[225,225,200,200,275,175]$} \\
MTP-5 & $c_{i j}=[10182 ; 9820 ; 14217 ; 12225], s_{i j}=[500,250,350,600], d_{i j}=[300,600,800]$ \\
\hline
\end{tabular}




\section{Appendix D}

\begin{tabular}{ll}
\hline Problems & Data of the problems \\
\hline BTP-1 (Ekanayake, 2020) & $c_{i j}=[6,8,10 ; 7,11,11 ; 4,5,12], s_{i j}=[150,175,275], d_{i j}=[200,100,300]$ \\
BTP-2 (Ahamad, 2017) & $c_{i j}=[15,7,25 ; 8,12,14 ; 17,19,21], s_{i j}=[12,17,7], d_{i j}=[12,10,14]$ \\
BTP-3 (Khan, 2015) & $c_{i j}=[3,6,8,4 ; 6,1,2,5 ; 7,8,3,9], s_{i j}=[13,28,17], d_{i j}=[15,19,13,18]$ \\
BTP-4 (Ekanayake, 2020) & $c_{i j}=[4,6,8,8 ; 6,8,6,7 ; 5,7,6,8], s_{i j}=[40,60,50], d_{i j}=[20,30,50,50]$ \\
BTP-5 (Ahamad, 2017) & $c_{i j}=[5,7,10,5,3 ; 8,6,9,12,14 ; 10,9,8,10,15], s_{i j}=[5,10,10], d_{i j}=[3,3,10,5,4]$ \\
BTP-6 (Deshmukh, 2012) & $c_{i j}=[4,1,2,4,4 ; 2,3,2,2,3 ; 3,5,3,4,4], s_{i j}=[60,35,40], d_{i j}=[22,45,20,18,30]$ \\
BTP-7 (Ekanayake, 2020) & $c_{i j}=[7,5,9,11 ; 4,3,8,6 ; 3,8,10,5 ; 2,6,7,3], s_{i j}=[30,20,25,15], d_{i j}=[30,30,20,10]$ \\
& $c_{i j}=[25,14,34,46,45 ; 10,47,14,20,41 ; 22,42,38,21,46 ; 36,20,41,38,44], s_{i j}=$ \\
BTP-8 (Ekanayake, 2020) & {$[27,35,37,45], d_{i j}=[22,27,28,33,34]$} \\
BTP-9 (Khan, 2015) & $c_{i j}=\left[7,10,7,4,78 ; 5,1,5,5,3,3 ; 4,3,7,9,1,9[4,6,9,0,0,8], s_{i j}=[5,6,2,9], d_{i j}=[4,4,6,2,4,2]\right.$ \\
& $c_{i j}=[73,40,9,79,20 ; 62,93,96,8,13 ; 96,65,80,50,65,57,58,29,12,87 ; 56,23,87,18,12], s_{i j}=$ \\
BTP-10 (Uddin, 2016) & {$[8,7,9,3,5], d_{i j}=[6,8,10,4,4]$} \\
\hline
\end{tabular}

\section{Appendix E}

\section{Problems Data of the problems}

UTP-1 (Girmay, 2013) $\quad c_{i j}=[4,8,8 ; 16,24,16 ; 8,16,24], s_{i j}=[76,82,77], d_{i j}=[72,102,44]$

UTP-2 (Ahamad, 2017) $\quad c_{i j}=[10843 ; 1214202 ; 692325], s_{i j}=[500,400,300], d_{i j}=[250,350,600,150]$

UTP-3 (Ahamad, 2016) $\quad c_{i j}=[1210613 ; 1981625 ; 17151520 ; 23222612], s_{i j}=[150,200,600,225]$,

$$
d_{i j}=[300,500,75,100]
$$

UTP-4 (Ahamad, 2016) $\quad c_{i j}=[58663 ; 47765 ; 84664], s_{i j}=[800,500,900], d_{i j}=[400,400,500,400,800]$

UTP-5 (Khan, 2015) $\quad c_{i j}=[54865 ; 45432 ; 36584], s_{i j}=[600,400,1000], d_{i j}=[450,400,200,250,300]$

UTP-6 (Khan, 2015) $\quad c_{i j}=[4,5,6, ; 3,1,5 ; 2,4,4], s_{i j}=[12,11,7], d_{i j}=[6,5,8]$

UTP-7 (Khan, 2015) $\quad c_{i j}=[25,17,25,14 ; 15,10,18,24 ; 16,20,8,13], s_{i j}=[300,500,600], d_{i j}=[300,300,500,500]$

UTP-8 (Ekanayake, $\quad c_{i j}=[5,4,8,6,5 ; 4,5,4,3,2 ; 3,6,5,84], s_{i j}=[600,400,1000], d_{i j}=[450,400,200,250,300]$ 2020)

$\begin{array}{ll} & c_{i j}=[10,2,16,14,10 ; 6,18,12,13,16 ; 8,4,14,12,10 ; 14 \\ \text { UTP-9 (Khan, 2015) } & {[300,500,825,375], d_{i j}=[350,400,250,150,100]}\end{array}$

UTP-10 (Uddin, 2016) $\quad c_{i j}=[42,48,38,37 ; 40,49,52,51 ; 39,38,40,43],, s_{i j}=[160,159,190], d_{i j}=[80,90,110,160]$

\section{Copyrights}

Copyright for this article is retained by the author(s), with first publication rights granted to the journal.

This is an open-access article distributed under the terms and conditions of the Creative Commons Attribution license (http://creativecommons.org/licenses/by/4.0/). 